\title{
Extended Kalman Filtering with Nonlinear Equality Constraints: a Geometric Approach
}

\author{
Axel Barrau, Silvère Bonnabel *
}

\begin{abstract}
In this paper, we focus on extended Kalman filtering (EKF), in the difficult case where a function of the state has been perfectly observed, and is thus known with certainty, while the full state still has unobserved degrees of freedom. In the linear case, the Kalman filter seamlessly handles such constraints, which result in the state being in an affine subspace. Yet, in the nonlinear case, the EKF poorly handles such type of constraints. As a remedy, we propose a novel general methodology of EKF based on an (arbitrary) nonlinear error $e$. And we prove that under compatibility of the error $e$ with the constraints, the EKF based on $e$ seamlessly handles the constraints. Furthermore, when the state space is a Lie group, we prove the EKF based on invariant errors is exactly the invariant EKF (IEKF), and we prove further properties. The theory is applied to the problem of simultaneous localization and mapping (SLAM), where the IEKF is shown to perfectly handle some partial deterministic information about the map. As a byproduct, the theory is also shown to readily allow devising EKFs on state spaces defined by a class of equality constraints.
\end{abstract}

\section{Introduction}

The extended Kalman filter (EKF) is an estimation algorithm that is pervasively used in various fields involving dynamical systems' state estimation from measurements of physical sensors. When the considered system is linear and the uncertainties are modeled by Gaussian random variables, the Kalman filter (KF) is optimal. On the other hand, when the system is nonlinear the EKF relies on first-order Taylor expansion of the nonlinear system, and it is not optimal.

It is common to use the EKF in contexts where some side (deterministic) information about the state is available. This has led to the realm of state constrained extended Kalman filtering, see e.g. [24], where a variety of tools allows forcing the state to belong to a set where it is known to be: for instance concentrations in chemical reactors are

${ }^{*}$ A. Barrau is with SAFRAN TECH, Groupe Safran, Rue des Jeunes Bois - Châteaufort, 78772 Magny Les Hameaux CEDEX, France axel. barrau@safran.fr. S. Bonnabel is with MINES ParisTech, PSL Research University, Centre for robotics, 60 Bd St Michel 75006 Paris, France and with Université de la Nouvelle-Calédonie, Institut de Sciences Exactes et Appliquées, 98851 Nouméa Cedex. silvere.bonnabel@mines-paristech.fr 
positive. More recently, some techniques have also emerged in the domain of optimization based Kalman smoothing [7].

In this paper, we consider a class of constraints poorly handled by the EKF, and remedy its problems by introducing a general methodology of EKF based on a nonlinear error $e$ (see Section 1.1). Furthermore, when the state space is a Lie group we relate the proposed methodology to the invariant EKF (IEKF) of [3]. Building upon Lie group theory, and notably the Lie group - Lie algebra correspondance, we show stronger results may be obtained for the Lie group case.

The starting point of our study was the recent successful implementation of the IEKF in an industrial product for high precision navigation described in [6], where a high end inertial measurement unit yields increments of such precision that the dynamics is almost noise free, and where in contrast, the initial heading of the vehicle is totally unknown, yielding a (theoretical) covariance matrix with a mixture of large eigenvalues and very small eigenvalues. For this problem the IEKF was found to outperform the EKF so univocally that strong underlying theoretical properties could be suspected.

Partial explanations can be found in the preliminary conference paper [2], where a simplified example of high precision navigation was considered, which is recalled in Figure 1. In a following preliminary conference paper [12], we proved some properties of the IEKF regarding state constraints for the particular class of mixed-invariant noise free dynamics on Lie groups, see e.g., [5,19].

The constraints we consider are technically related to rank deficiency of the EKF's covariance matrix. To this respect, we would like to mention that KF and EKF with rank deficient covariance matrices have been the object of recent research in atmospheric and oceanographic contexts, where filtering and smoothing are referred to as data assimilation, and where the dynamical models are based on fluid mechanics described by partial differential equations, and their discretization results in high dimensional state spaces that make Kalman filtering intractable. Yet, there has been evidence that Kalman filtering with reduced dimensional covariance matrices could prove sufficient. Mathematical analysis of the linear KF with rank deficient covariance matrices is the subject of ongoing research, see e.g., $[8,16]$.

\subsection{The contribution simply explained}

Assume at some point the constraint ${ }^{1}$ is $h(X)=c$. Consider an EKF, and pick an estimate $\hat{X}$ that verifies $h(\hat{X})=c$. To make sure the covariance matrix also reflects this information, that is, all the uncertainty must be within the subset defined by $h(X)=c$, we make a virtual noise-free observation $Y=h(X)$ where we set $Y=c$ to update the EKF. The fact that $h(X)=c$ is now supposedly known for certain. However, if we immediately make a number of other observations $Y_{1}=h_{1}(X)+V_{1}, \cdots, Y_{n}=h_{n}(X)+V_{n}$, with $V_{i}$ 's independent noises, and denote $\hat{X}^{+}$the EKF's estimate taking into account all the latter measurements, do we have $h\left(\hat{X}^{+}\right)=c$ ? If $h$ is linear, then yes, the EKF does behave as if it had encoded $h(X)=c$ as a "hard constraint". But if $h$ is nonlinear, then unfortunately the answer is no.

In this paper we first advocate that if $e$ is a nonlinear estimation error of the general form $e=\Pi(\hat{X}, X)$, which offers an alternative to the usual linear state error $X-\hat{X}$,

\footnotetext{
${ }^{1}$ in the paper we rather use notation $q(X)=c$ in order not to confuse $q$ with the observation map $h$.
} 
an alternative EKF may in turn be built based on this estimation error: the covariance matrix then reflects the statistical dispersion of $e$ instead of the one of $X-\hat{X}$. Then, suppose we have a constraint of the form $h(X)=c$, and we apply the same procedure as above to an EKF based on error e. We pick an estimate such that $h(\hat{X})=c$, then update the EKF with the noise-free virtual observation $h(X)=c$, and then consider the updated state $\hat{X}^{+}$after additional observations $Y_{1}, \cdots, Y_{n}$. Then, the desirable condition $h\left(\hat{X}^{+}\right)=c$, is automatically satisfied under the following very simple condition: the kernel of $\left.\frac{\partial h}{\partial e}\right|_{X}$ viewed $^{2}$ as a function of $X$, is a function of the components of $h(X)$ only, i.e., does not fully depend on $X$.

For example, the conventional EKF is based on linear error $e=\Pi(\hat{X}, X)=X-\hat{X}$. If $h(X)=H X$ is linear, we have $\frac{\partial h}{\partial X}=H$. Thus $\frac{\partial h}{\partial e}=\frac{\partial h}{\partial X} \frac{\partial X}{\partial e}=\frac{\partial h}{\partial X}\left(\frac{\partial e}{\partial X}\right)^{-1}=H * I^{-1}=H$ and $\operatorname{Ker} \frac{\partial h}{\partial e}=\{z \mid H z=0\}$. As this subspace does not even depend on $X$, the condition is trivially met, and the theory confirms the EKF has a desirable behavior with respect to linear observations.

\subsection{Organization of the paper}

The linear case is studied in Section 2. The nonlinear case and the caveats of the EKF are described in Section 3. In Section 4, we introduce the novel general methodology of EKFs based on nonlinear errors, and prove it may remedy the problems of the EKF with respect to the considered class of constraints. The theory is then applied to the case where the state space is a (matrix) Lie group $G$, where we recover the IEKF of [3]. The obtained EKF not only keeps the state inside an embedded matrix Lie group, as customary in Bayesian filtering on Lie groups, e.g., $[9,10,15]$, but we obtain powerful results in Section 5 for problems which involve additional constraints that the state shall belong to a subgroup $G_{t}$ of $G$, that moreover may change over time. This is true even if the constraint appears during the experiment due to an observation, and without ad-hoc adaptation of the algorithm. Finally, in Section 6, the theory is applied to the problem of simultaneous localization and mapping (SLAM), see e.g. [14], where we consider a type of constraint that has never been explored before, and that is violated by the EKF. Although the paper is self-contained, the reader is advised to read [2] as an introduction.

\section{The linear case}

We recall in this section a known property of linear Kalman filtering regarding its ability to handle partially deterministic information in the absence of process noise.

\subsection{Considered problem}

Consider a continuous-discrete linear system in $\mathbb{R}^{d}$ with deterministic dynamics:

$$
\begin{aligned}
\frac{d}{d t} X_{t} & =A_{t} X_{t}+B u_{t}, \\
Y_{n} & =H_{n} X_{t_{n}}+V_{n},
\end{aligned}
$$

\footnotetext{
$\left.{ }^{2} \frac{\partial h}{\partial e}\right|_{X}$ is rigorously defined in the paper as $\left.\frac{\partial h}{\partial e}\right|_{X}=\frac{\partial h}{\partial X} \frac{\partial X}{\partial e}:=\left.\frac{\partial h}{\partial X}\right|_{X}(D \Pi(X))^{-1}$ with $D \Pi(X):=$ $\left.\frac{\partial}{\partial X} \Pi(\hat{X}, X)\right|_{(X, X)}$ the partial derivative of $e=\Pi(\cdot, \cdot)$ w.r.t. the second variable at $(X, X)$.
} 
where $X_{t} \in \mathbb{R}^{p}$ is the state of the system, $u_{t}$ a known deterministic input, $\left(t_{n}\right)_{n \geq 0}$ the sequence of observation times, $\left(Y_{n}\right)_{n \geq 0}$ the corresponding observations and $V_{n}$ a centered Gaussian noise with covariance matrix $R_{n}$ (assumed positive definite) corrupting $n$-th observation.

We also make the additional assumption that the initial distribution of the state lies in an affine subspace, that is:

$$
X_{0} \sim \mathcal{N}\left(\hat{X}_{0}, P_{0}\right), \quad \text { where } P_{0} \text { has rank } k<d .
$$

Definition 1 (prior distribution). Combining the initial distribution given by (3) and the dynamics (1), one gets a probability distribution $\pi\left(d x_{t}\right)$ of the state $X_{t}$ at all times $t \geq 0$. This is called the prior on the state $X_{t}$ and represents the information without the observations.

Bayesian filtering consists in computing in real time the statistics of the unknown state $X_{t}$ given all the information $y_{0}, y_{1}, \cdots, y_{n}$ up to present time $t$.

Definition 2 (posterior distribution). The posterior distribution of $X_{t}$ is the prior conditioned on past measurements, that is, the probability measure $\pi\left(d x_{t} \mid y_{0}, y_{1}, \cdots, y_{n}\right)$, where $t_{n} \leq t<t_{n+1}$.

Definition 3 (optimal filter). A filter that would be able to compute at all times the posterior distribution conditioned on the past is called the optimal filter.

\subsection{Support of the posterior distribution}

Definition 4 (Support of a distribution). If $X$ is a random variable in $\mathbb{R}^{d}$, the support of the distribution is the smallest closed set $W \subset \mathbb{R}^{d}$ such that we have $(X \in W)$ with probability 1, i.e., the set where $X$ lives.

Owing to (1)-(3), support of the posterior is restricted to an affine subspace of $\mathbb{R}^{d}$. Indeed, let $W_{0}$ denote the affine space $\hat{X}_{0}+\operatorname{Im} P_{0}$ : it has dimension $k$, and let $\vec{W}_{0}=\operatorname{Im} P_{0}$ be the associated vector space. We have:

Proposition 1. Let $F_{t} \in \mathbb{R}^{d \times d}$ be defined through $\frac{d}{d t} F_{t}=A_{t} F_{t}$ where $F_{0}=I$, and $Z_{t} \in \mathbb{R}^{d}$ be defined by $\frac{d}{d t} Z_{t}=A_{t} Z_{t}+B u_{t}$ with $Z_{0}$ any element of $W_{0}$. At time $t$, the support of the prior distribution is $W_{t}=Z_{t}+\vec{W}_{t}$, where $\vec{W}_{t}=F_{t} \vec{W}_{t}$. Thus, we see the prior at any time is also supported by an affine subspace $W_{t}$ of $\mathbb{R}^{d}$.

Proof. At time $t \geq 0$, the distribution of $X_{t}$ is supported by the image of $W_{0}$ through the linear equation (1), and we see $X_{t}-Z_{t}=F_{t}\left(X_{0}-Z_{0}\right)$.

Proposition 2. From the definitions of conditional probability, we see the support of the posterior distribution is contained in the support of the prior. Thus Proposition 1 implies that at time $t \geq 0$ the distribution computed by the optimal filter is supported by $W_{t}=Z_{t}+\vec{W}_{t}$. 


\subsection{Support of the Kalman filter's belief}

Definition 5. For any (not necessary optimal) filter, we call belief the posterior distribution output by the filter.

As the Kalman filter (KF) is the optimal filter for the linear-Gaussian systems of the form (1)-(2), its belief is necessarily supported by the affine subspace $W_{t}=Z_{t}+\vec{W}_{t}$. However, it is instructive to see how the KF actually maintains the belief in $W_{t}$.

\subsubsection{Kalman filter's equations}

At each time the KF's belief is as follows $X_{t} \sim \mathcal{N}\left(\hat{X}_{t}, P_{t}\right)$ where $\hat{X}_{t}, P_{t}$ are defined through an alternation between propagation and update steps.

- Initialization: The initial belief is $X_{0} \sim \mathcal{N}\left(\hat{X}_{0}, P_{0}\right)$.

- Propagation step: Between two measurements $t_{n-1} \leq t<t_{n}$, the belief is propagated as:

$$
\frac{d}{d t} \hat{X}_{t}=A_{t} \hat{X}_{t}+B u_{t}, \frac{d}{d t} P_{t}=A_{t} P_{t}+P_{t} A_{t}^{T}
$$

- Update step: When a measurement arrives at time $t_{n}$ the belief is updated as $X_{t_{n}} \sim$ $\mathcal{N}\left(\hat{X}_{t_{n}}^{+}, P_{t_{n}}^{+}\right)$where

$$
\begin{aligned}
z_{n} & :=Y_{n}-H_{n} \hat{X}_{t_{n}}, \\
K_{n} & :=P_{t_{n}} H_{n}^{T}\left(H_{n} P_{t_{n}} H_{n}^{T}+R_{n}\right)^{-1}, \\
\hat{X}_{t_{n}}^{+} & =\hat{X}_{t_{n}}+K_{n} z_{n}, \quad P_{t_{n}}^{+}=\left(I-K_{n} H_{n}\right) P_{t_{n}},
\end{aligned}
$$

and where $z_{n}$ is called the innovation.

\subsubsection{Support of the belief}

We can prove:

Proposition 3. The support of the Kalman Filter's belief is $W_{t}$, i.e., at all times we have $\hat{X}_{t}+\operatorname{Im} P_{t}=W_{t}$.

Proof. From Proposition 1 , we may take $Z_{t}:=\hat{X}_{t}$ and all we have to prove is $\operatorname{Im} P_{t}=$ $\vec{W}_{t}$. By induction: assume $\operatorname{Im} P_{t_{n-1}}=\overrightarrow{W_{t_{n-1}}}$. Pick arbitrary $\tilde{x}_{t_{n-1}} \in \mathbb{R}^{d}$. We have $\left[P_{t_{n-1}} \tilde{x}_{t_{n-1}}\right] \in \operatorname{Im} P_{t_{n-1}}=\overrightarrow{W_{t_{n-1}}}$. For $t \geq t_{n-1}$ let $\tilde{x}_{t}$ satisfy the equation $\frac{d}{d t} \tilde{x}_{t}=-A_{t}^{T} \tilde{x}_{t}$. Using (4) we have $\frac{d}{d t}\left[P_{t} \tilde{x}_{t}\right]=A_{t}\left[P_{t} \tilde{x}_{t}\right]+P_{t} A_{t}^{T} \tilde{x}_{t}-P_{t} A_{t}^{T} \tilde{x}_{t}=A_{t}\left[P_{t} \tilde{x}_{t}\right]$. Thus $\operatorname{Im} P_{t}=\overrightarrow{W_{t}}$ owing to definition of $F_{t}$ in Proposition 1, implying $\operatorname{Im} P_{t_{n}}=\overrightarrow{W_{t_{n}}}$. At the update, $\operatorname{Im} K_{n} \subset \operatorname{Im} P_{t_{n}}$ from (6) implying that $\hat{X}_{t_{n}}^{+}=\hat{X}_{t_{n}}+K_{n} z_{n} \in W_{t_{n}}$, and that $\operatorname{Im} P_{t_{n}}^{+} \subset \overrightarrow{W_{t_{n}}}$ since $P_{t_{n}}^{+}=P_{t_{n}}-K_{n} H_{n} P_{t_{n}}$. This proves $\hat{X}_{t_{n}}^{+}+\operatorname{Im} P_{t_{n}}^{+}=W_{t_{n}}$.

\section{The nonlinear case}

In this section, we discuss the case where the initial covariance matrix is singular and the dynamics deterministic but nonlinear. We also evidence the caveats of rank-deficient extended Kalman filtering. 


\subsection{Considered problem}

Consider the nonlinear system in $\mathbb{R}^{d}$ :

$$
\begin{aligned}
\frac{d}{d t} X_{t} & =f\left(X_{t}, u_{t}\right), \\
Y_{n} & =h\left(X_{t_{n}}\right)+V_{n},
\end{aligned}
$$

where $f, h$ are smooth nonlinear maps, $u_{t}$ a deterministic and fully known input, and $V_{n} \sim \mathcal{N}\left(0, R_{n}\right)$. Assume as previously that the initial distribution of the state lies in an affine subspace, i.e.,

$$
X_{0} \sim \mathcal{N}\left(\hat{X}_{0}, P_{0}\right), \quad \text { where } P_{0} \text { has rank } k<d .
$$

Definition 6. Denote by $\Psi(t ; x)$ the solution of equation (8) at time $t$ when initialized on $x . \Psi$ is called the flow of the equation. It is characterized by $\frac{d}{d t} \Psi(t ; x)=f(\Psi(t ; x), t)$ with $\Psi(0 ; x)=x$.

Note that, in the linear case (1) of the preceding section, we have $\Psi(t ; x)=F_{t} x$, see Proposition 1.

Remark 1. More generally, we can assume that the initial distribution is supported by some manifold $\mathcal{M}_{0}$, and all the developments to come remain perfectly valid. However, the present section is concerned with initial prior (10) for simplicity of exposition.

\subsection{Support of the posterior distribution}

Owing to nonlinear dynamics (8), the initial affine subspace gets distorted over time.

Proposition 4. The image of the affine subspace $W_{0}=\hat{X}_{0}+\operatorname{Im} P_{0}$ through the flow $\Psi$ of (8) defines a submanifold $\mathcal{M}_{t}=\left\{\Psi(t ; x) \mid x \in W_{0}\right\}$ of $\mathbb{R}^{d}$ of dimension $k$. Moreover, the tangent space $T_{\Psi(t ; x)} \mathcal{M}_{t}$ of $\mathcal{M}_{t}$ at $\Psi(t ; x)$ is given by the partial derivatives of $\Psi(t ; x)$ with respect to $x$ in directions defined by $\vec{W}_{0}$.

More precisely we have:

Proposition 5. Consider a solution $\left(X_{t}\right)_{t \geq 0}$ of (8), with $X_{0}=x_{0}$. At $X_{t}=\Psi\left(t ; x_{0}\right)$, the tangent space to $\mathcal{M}_{t}$ is spanned by the columns of a matrix $M\left(\Psi\left(t ; x_{0}\right), t\right) \in \mathbb{R}^{d \times k}$, which satisfies the equation $\frac{d}{d t} M=\frac{\partial f}{\partial x} M$ with initial condition $\operatorname{Im} M\left(x_{0}, 0\right)=\vec{W}_{0}$.

Proof. As $f$ is smooth so is $\Psi$. Denote $\partial_{\bar{x}} \Psi\left(t ; x_{0}\right)$ the partial derivative in the direction of $\bar{x} \in \mathbb{R}^{d}$. We have $\partial_{t}\left(\partial_{\bar{x}} \Psi\left(t ; x_{0}\right)\right)=\partial_{\bar{x}} \partial_{t} \Psi\left(t ; x_{0}\right)=\partial_{\bar{x}} f\left(\Psi\left(t ; x_{0}\right), t\right)=\frac{\partial f}{\partial x}\left(\Psi\left(t ; x_{0}\right), t\right) \partial_{\bar{x}} \Psi\left(t ; x_{0}\right)$. Using a basis $\bar{x}_{1}, \cdots, \bar{x}_{d}$ and the corresponding vectors $\partial_{\bar{x}_{i}} \Psi\left(t ; x_{0}\right) \in \mathbb{R}^{d}$ as columns of a matrix $N_{t}$ we have proved $\dot{N}_{t}=\frac{\partial f}{\partial x}\left(\Psi\left(t ; x_{0}\right), t\right) N_{t}$. From Liouville's formula (Lemma 3.11 of [26] $) \operatorname{det} N_{t}=\operatorname{det} N_{0} \exp \left(\int_{0}^{t} \operatorname{tr}\left(\frac{\partial f}{\partial x}\left(\Psi\left(s ; x_{0}\right), s\right)\right) d s\right)$ and thus $N_{t}$ is invertible and thus so is the differential of $\Psi$. Thus $W_{0}$ provides a set of local coordinates on the set $\mathcal{M}_{t}=\left\{\Psi(t ; x) \mid x \in W_{0}\right\}$ through $\Psi(t ; \cdot)$. This proves $\mathcal{M}_{t}$ is a submanifold of dimension $k=\operatorname{dim} W_{0}$. It also proves $\partial_{\bar{x}} \Psi(t ; x)$ is a tangent vector of $\mathcal{M}_{t}$ at $\Psi(t ; x)$ for $\bar{x} \in \vec{W}_{0}$. Similarly, the set $\left\{\partial_{\bar{x}} \Psi\left(t ; x_{0}\right) \mid \bar{x} \in \vec{W}_{0}\right\}$ is spanned by the columns of a matrix $M_{t} \in \mathbb{R}^{d \times k}$ solution to $\frac{d}{d t} M_{t}=\frac{\partial f}{\partial x} M_{t}$ with initial condition $\operatorname{Im} M_{0}=\overrightarrow{W_{0}}$, and naturally $\operatorname{Im} M\left(\Psi\left(t ; x_{0}\right), t\right)=T_{\Psi\left(t ; x_{0}\right)} \mathcal{M}_{t}$. 
Note that, $\left(X_{t}, M\right)$ may in fact be defined intrinsically through the notion of variational system as introduced in [13]. Proposition 4 implies the prior distribution is supported by $\mathcal{M}_{t}$. From the definitions of conditional probability, we have the following result which is the nonlinear counterpart of Proposition 2.

Proposition 6. The posterior distribution is supported at all times by the $k$-dimensional manifold $\mathcal{M}_{t}$. In other terms, $\mathcal{M}_{t}$ supports the belief of the optimal filter.

\subsection{Support of the extended Kalman filter belief}

The extended Kalman filter (EKF), is an extension of the KF to the nonlinear case, based upon linearizations. Its very "linear" structure, turns out to be inappropriate in the context of nonlinear dynamics along with singular covariance matrices. The reasons why are as follows.

\subsubsection{Extended Kalman filter's equations}

At each time the EKF's belief $X_{t} \sim \mathcal{N}\left(\hat{X}_{t}, P_{t}\right)$ is defined through an alternation of propagation and update:

- Initialization: The initial belief is $X_{0} \sim \mathcal{N}\left(\hat{X}_{0}, P_{0}\right)$.

- Propagation step: Between two measurements $t_{n-1} \leq t<t_{n}$, the belief is propagated as follows:

$$
\frac{d}{d t} \hat{X}_{t}=f\left(\hat{X}_{t}, u_{t}\right), \quad \frac{d}{d t} P_{t}=A_{t} P_{t}+P_{t} A_{t}^{T},
$$

where $A_{t}=\frac{\partial f}{\partial x}\left(\hat{X}_{t}, u_{t}\right)$.

- Update step: When a measurement arrives at time $t_{n}$ the belief is updated as $X_{t_{n}} \sim$ $\mathcal{N}\left(\hat{X}_{t_{n}}^{+}, P_{t_{n}}^{+}\right)$where

$$
\begin{aligned}
z_{n} & :=Y_{n}-\hat{X}_{t_{n}}, \quad H_{n}:=\frac{\partial h}{\partial x}\left(\hat{X}_{t_{n}}\right) \\
K_{n} & :=P_{t_{n}} H_{n}^{T}\left(H_{n} P_{t_{n}} H_{n}^{T}+R_{n}\right)^{-1}, \\
\hat{X}_{t_{n}}^{+} & =\hat{X}_{t_{n}}+K_{n} z_{n}, \quad P_{t_{n}}^{+}=\left(I-K_{n} H_{n}\right) P_{t_{n}} .
\end{aligned}
$$

\subsubsection{Support of the belief}

First of all, as the EKF represents the belief as a Gaussian, it is hopeless that the support of the belief match with $\mathcal{M}_{t}$, since the latter is a curved space whereas Gaussians are supported by affine spaces (in other words the EKF computes linear Gaussian approximations to the posterior). However, what one may desire is that belief distribution $\mathcal{N}\left(\hat{X}, P_{t}\right)$ of the EKF be consistent with the geometric constraints.

Definition 7. We say that the EKF (or any Gaussian filter) belief $\mathcal{N}\left(\hat{X}_{t}, P_{t}\right)$ is consistent with the state space constraint $\mathcal{M}_{t}$ if at all times $t \geq 0$ we have necessarily $1-\hat{X}_{t} \in \mathcal{M}_{t}$, and 2- $\operatorname{Im} P_{t}=T_{\hat{X}_{t}} \mathcal{M}_{t}$. In other words, the support of the distribution $\mathcal{N}\left(\hat{X}_{t}, P_{t}\right)$ must coincide with the tangent space $T_{\hat{X}_{t}} \mathcal{M}_{t}$. 
Unfortunately, when the constraints are induced by initial rank deficient prior covariance matrix and the dynamics nonlinear, Definition 7 is never met by the EKF, as a consequence of its "linear" structure. Indeed, to meet conditions 1 and 2 of Definition 7 at all times, both propagation and update steps must preserve those conditions. Propagation step is not problematic in itself:

Proposition 7. During the propagation step, the EKF preserves conditions 1 and 2.

Proof. First of all, it is clear that $\hat{X}_{t}$ will remain in $\mathcal{M}_{t}$ during propagation since the EKF merely integrates the model equations, see (11). Between two updates, we see from Proposition 5 that $T_{\hat{X}} \mathcal{M}_{t}$ is spanned by a matrix $M_{t}$ which is a solution to the equation $\frac{d}{d t} M_{t}=A_{t} M_{t}$. Thus as shown in the proof of Proposition 3 , if at some point $\operatorname{Im} P_{t}=\operatorname{Im} M_{t}$ this will remain true in the absence of updates.

On the other hand, the update step is problematic for the reasons to follow. To provide a more concrete picture, we have illustrated the following items using an example of engineering interest discussed in the preliminary conference paper [2], please see Figure 1 .

- Assume that just before update at time $t_{n}$ the EKF is consistent with the constraint and meets conditions 1 and 2 of Definition 7.

- Condition 1: as $\operatorname{Im} P_{t_{n}}=T_{\hat{X}_{t_{n}}} \mathcal{M}_{t_{n}}$ the correction term $K_{n} z_{n}$ points in a constraintconsistent direction, i.e., $K_{n} z_{n} \in T_{\hat{X}_{t_{n}}} \mathcal{M}_{t_{n}}$. But then the updated state $\hat{X}_{t_{n}}^{+}=$ $\hat{X}_{t_{n}}+K_{n} z_{n}$ is generally not an element of $\mathcal{M}_{t_{n}}$ since $\mathcal{M}_{t_{n}}$ is not a vector space, see Figure 1 (b).

- Condition 2: even if $\hat{X}_{t_{n}}^{+} \in \mathcal{M}_{t_{n}}$, condition 2 will not be met since, owing to the update step, we have also $\operatorname{Im} P_{t_{n}}^{+} \subset T_{\hat{X}_{t_{n}}} \mathcal{M}_{t_{n}}$, and there is no reason why $T_{\hat{X}_{t_{n}}} \mathcal{M}_{t_{n}}$ should coincide with $T_{\hat{X}_{t_{n}}^{+}} \mathcal{M}_{t_{n}}$, the manifold $\mathcal{M}_{t_{n}}$ being curved, see Fig. 1 (c).

\subsubsection{Discussion on state constrained based solutions}

One possible solution is to consider that the state be part of $\mathcal{M}_{t}$ as a state constraint. For a review on state constrained EKFs see e.g. [23]. The present article aims at providing a geometric framework that offers alternatives to this route. Indeed, contrary to state constrained solutions, the solutions presented in the remainder of the paper do not require specific adaptation to the particular constraints considered so that first-order optimality properties are conserved, as the EKF's outputs are never transformed nor projected in order to artificially enforce the constraint. Moreover, the constraints considered in the present paper are time-varying, which is a further challenge. Besides, our framework becomes especially powerful when soft contraints such as "the car is close to the circle" are created by the observations, please see the paper's conclusion. 


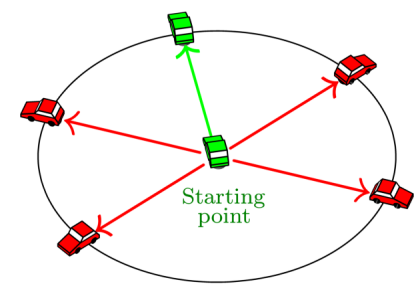

(a)

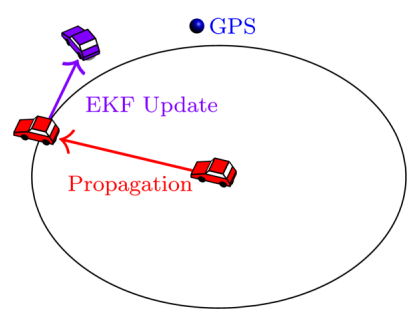

(b)

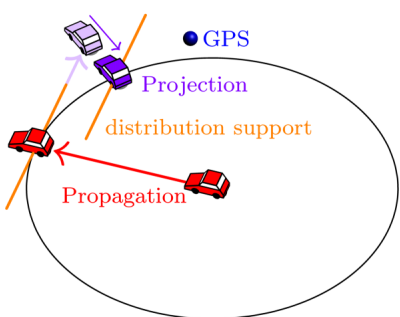

(c)

Figure 1: Consider a simplified car in 2D, equipped with perfect differential wheel speeds, and a GPS that returns discrete noisy measurements of the position. (a) The (green) car starts from perfectly known initial position with unknown heading, and wheel speeds inform the filter that the car drives in straight (green) line until time $t>0$. It is thus known to be located with certainty on a circle centered at the initial position, but at an unknown location (all red cars are possible locations): there is uncertainty along the circle, and certainty orthogonally to it. (b) An EKF may be used to estimate the position and the orientation. Suppose at $t$, a GPS measurement is available and must be combined with current knowledge about the state. To do so, the EKF corrects the state, by moving the position in the direction of the measurement. It locally uses the information the car should be on the circle, and thus computes a correction term which is tangential to the circle. But due to the "linear" structure of the filter, adding the computed correction to update the state results in an updated position that lies out of the circle. (c) Moreover, starting with a belief indicating uncertainty in the direction tangential to the circle, even if the updated state is artificially projected onto the circle, the new belief of the EKF becomes transverse to the circle. This is due to the image of covariance matrix after the update being always included in the image of the covariance matrix before the update. 


\section{A general approach: EKF based on nonlinear er- rors}

The problem of the EKF is its linear structure, that does not match with the true dispersion of the belief inside a curved space $\mathcal{M}_{t}$. The estimation error shall increase along $\mathcal{M}_{t}$ indeed, but never transversally to it.

\subsection{EKF based on an alternative error}

Instead of considering the standard estimation error $\hat{X}_{t}-X_{t}$, we propose to define an alternative nonlinear estimation error as $e_{t}=\Pi\left(\hat{X}_{t}, X_{t}\right)$, with $\Pi: \mathbb{R}^{d} \times \mathbb{R}^{d} \rightarrow \mathbb{R}^{d}$ a function that satisfies $\Pi(x, x) \equiv 0$, and which is locally invertible with respect to both of its arguments at the diagonal set $\left\{(x, x) \mid x \in \mathbb{R}^{d}\right\}$. A Taylor expansion of the nonlinear error yields $\Pi(\hat{X}, \hat{X}+\delta X)=D \Pi(\hat{X}) \delta X+O\left(\|\delta X\|^{2}\right)$. The operator $D \Pi$ is everywhere invertible owing to the local invertibility property of $\Pi$ about the diagonal. It relates the error $e$ to the usual error $\delta X=X-\hat{X}$, and this may be memorized as

$$
\text { " } e=D \Pi(\hat{X}) \delta X " \quad \text { up to the first order. }
$$

An EKF based on this error works by letting $P_{t}^{e}$ reflect the local (linearized) dispersion of this alternative error. This naturally yields the following equations.

- Initialization: The initial belief is that $X_{0}$ has mean $\hat{X}_{0}$ and nonlinear error $e$ between $X_{0}$ and $\hat{X}_{0}$ is distributed as $e_{0} \sim \mathcal{N}\left(0, P_{0}^{e}\right)$.

- Propagation step: Between two measurements $t_{n-1} \leq t<t_{n}$, the belief is propagated as follows:

$$
\frac{d}{d t} \hat{X}_{t}=f\left(\hat{X}_{t}, u_{t}\right), \quad \frac{d}{d t} P_{t}^{e}=A_{t}^{e} P_{t}^{e}+P_{t}^{e}\left(A_{t}^{e}\right)^{T}
$$

where $A_{t}^{e}$ represents the first-order approximation to the error evolution, namely it is defined by

$$
\frac{d}{d t} e_{t}=A_{t}^{e} e_{t}+O\left(\left\|e_{t}\right\|^{2}\right)
$$

- Update step: When a measurement arrives at time $t_{n}$ the belief about state $X_{t_{n}}$ is updated as having mean $\hat{X}_{t_{n}}^{+}$, and error with respect to $\hat{X}_{t_{n}}^{+}$is updated as having distribution $e_{t_{n}}^{+} \sim \mathcal{N}\left(0,\left(P_{t_{n}}^{e}\right)^{+}\right)$, where we let

$$
\begin{gathered}
z_{n}:=Y_{n}-h\left(\hat{X}_{t_{n}}\right), \\
K_{n}:=P_{t_{n}}^{e}\left(H_{n}^{e}\right)^{T}\left(H_{n}^{e} P_{t_{n}}^{e}\left(H_{n}^{e}\right)^{T}+R_{n}\right)^{-1}, \\
\hat{X}_{t_{n}}^{+}=\varphi\left(\hat{X}_{t_{n}}, K_{n} z_{n}\right), \\
\left(P_{t_{n}}^{e}\right)^{+}=\left(I-K_{n} H_{n}^{e}\right) P_{t_{n}}^{e},
\end{gathered}
$$

and where $H_{n}^{e}$ is the linearized output map with respect to the error $e$, i.e., it is defined by

$$
z_{n}:=Y_{n}-h\left(\hat{X}_{t_{n}}\right)=H_{n}^{e} e_{t_{n}}+V_{n}+O\left(\left\|e_{t_{n}}\right\|^{2}\right),
$$

and where $\varphi$ is a function (we call exponential) to be introduced at Definition 8 . 


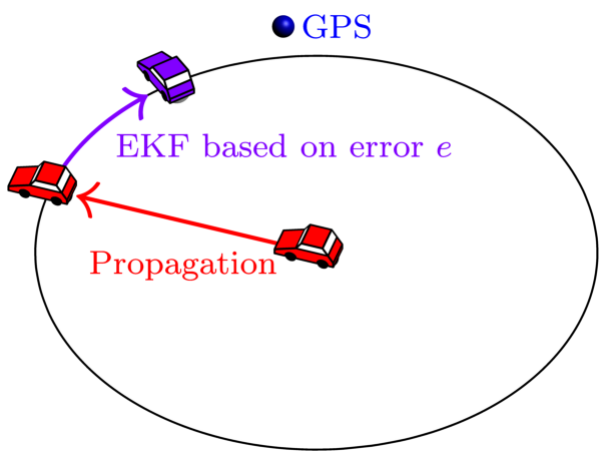

Figure 2: Same setting as Fig. 1, but where update is performed using nonlinear state error $e=\left(\hat{\theta}-\theta, R(\theta)^{T}(\hat{x}-x)\right)$ with $\theta$ the orientation of the car, $x \in \mathbb{R}^{2}$ its position, and $R(\theta)$ the rotation of angle $\theta$, see Section 6.1. We see the update follows the circle.

Note that, the practitioner may need to output the covariance in the original variables $X$. According to our belief that $e_{t} \sim \mathcal{N}\left(0, P_{t}^{e}\right)$, and owing to $(15)$, we may write $\operatorname{Cov}\left(X_{t}\right)=$ $D \Pi\left(\hat{X}_{t}\right)^{-1} P_{t}^{e}\left(D \Pi\left(\hat{X}_{t}\right)^{-1}\right)^{T}$. This will be more thoroughly proved and discussed at $(26)$.

Remark 2. Obviously $H_{n}^{e}=\frac{\partial h}{\partial X}\left(\hat{X}_{t_{n}}\right) D \Pi\left(\hat{X}_{t_{n}}\right)^{-1}$. It can be memorized as " $H_{n}^{e}:=\frac{\partial h}{\partial e}=$ $\frac{\partial h}{\partial X} \frac{\partial X}{\partial e}$ " and referring to (15), we see indeed " $\left(\frac{\partial X}{\partial e}\right)^{-1}=\frac{\partial e}{\partial X}=D \Pi "$.

$K_{n} z_{n}$ is a correction term to be applied to the state $\hat{X}_{t_{n}}$ in order to take into account the measurement $Y_{n}$. It defines a direction, in terms of the nonlinear error e, along which the estimate should be moved.

Definition 8. We define the function $\varphi$ in (20), that we call "exponential", as follows:

$$
\varphi(\hat{X}, \delta e):=\tilde{\chi}(1)
$$

where $\tilde{\chi}$ is defined as the solution of the differential equation:

$$
\frac{d}{d s} \tilde{\chi}(s)=(D \Pi(\tilde{\chi}(s)))^{-1} \delta e, \quad \tilde{\chi}(0)=\hat{X} .
$$

The rationale is as follows. Before the latest measurement $Y_{n}$, the EKF classically makes the approximation the average error between the true state $X_{t_{n}}$ and the predicted state $\hat{X}_{t_{n}}$ is null. The correction term $\delta e=K z$ is then a linear approximation to the (linear) Kalman filter estimate of the average error between the estimated and the true state, taking into account $Y_{n}$. As a result, the updated error must be equated with $\delta e$, at least up to the first order, that is, we could have picked any function $\varphi$ being such that $\hat{X}^{+}=\varphi(\hat{X}, \delta e) \Leftrightarrow \delta e=\Pi\left(\hat{X}, \hat{X}^{+}\right)$up to first order terms in $\delta e$. This is obviously the case with Definition 8 since $\varphi(\hat{X}, \delta e)=\hat{X}+(D \Pi(\hat{X}))^{-1} \delta e+O\left(\|\delta e\|^{2}\right)$. There are further reasons for using our "exponential", though, that will be made clear in Section 4.3 dealing with the constraints $\mathcal{M}_{t}$ we want to enforce, see Figure 2 for an illustration.

Remark 3. The conventional EKF of Section 3.3.1 is easily seen to be the EKF based on error $e=X-\hat{X}$.

Remark 4. In the considered problem, dynamics (8) are noise free. If they were corrupted by an additive white noise with covariance matrix $Q_{t}$, an EKF based on e could still be devised, but the right hand side of (16) should be replaced with $\frac{d}{d t} P_{t}^{e}=A_{t}^{e} P_{t}^{e}+P_{t}^{e}\left(A_{t}^{e}\right)^{T}+$ $D \Pi\left(\hat{X}_{t}\right) Q_{t} D \Pi\left(\hat{X}_{t}\right)^{T}$, see Section 4.4 for details. 


\subsection{Relation to changes of coordinates}

To gain insight into the choice of our exponential map (23), we first relate EKFs based on nonlinear errors to changes of coordinates. It can be proven inded that in the particular case where the nonlinear error $e$ corresponds to the linear error $Z-\hat{Z}$ in alternative coordinates $\phi(X)=Z$ with $\phi$ a nonlinear map, then the EKF based on error $e=$ $\Pi(\hat{X}, X):=Z-\hat{Z}=\phi(X)-\phi(\hat{X})$ in the sense of Section 4.1, and the conventional EKF built using directly the coordinates $Z$ coincide, as proved by Proposition 12 in Appendix A. In particular, the map $\varphi$ of Definition 8 then boils down to a mere vector addition in the $Z$ coordinates system, i.e., $\phi \circ \varphi(X, \delta e)=\phi(X)+\delta e$. This result offers insight into the interest of the method: if it turns out there exists alternative coordinates that make the constraints $\mathcal{M}_{t}$ linear subspaces, then one should definitely devise an EKF using these coordinates. And to some extent, this is the rationale we pursue in this paper. However, it should be noted that our method is broader, as EKFs based on nonlinear errors define a much larger class than EKFs devised in another coordinate system: we never required $\Pi(\hat{X}, X)$ to be of the form $\phi(X)-\phi(\hat{X})$. Notably, it may be proved there exists no alternative coordinate system in which the IEKF of Section 5 boils down to a conventional EKF built using those coordinates.

\subsection{Support of the belief of the EKF based on nonlinear esti- mation errors}

In this section, we are going to show how nonlinear errors may allow enforcing the constraint $\mathcal{M}_{t}$. To do so, we will assume that, 1) at each time $t$, there exists a submersion $q_{t}: \mathbb{R}^{d} \mapsto \mathbb{R}^{d-k}$, i.e., a map such that its differential $D q_{t}(x)$ has constant rank $d-k$ over $\mathbb{R}^{d}$, and that 2) the submanifold $\mathcal{M}_{t}$ is equal to $q_{t}^{-1}(c)=\left\{x \in \mathbb{R}^{d} \mid q_{t}(x)=c\right\}$ for some constant $c \in \mathbb{R}^{d-k}$; that is, $\mathcal{M}_{t}$ is an embedded submanifold that corresponds to a level set of $q_{t}$. Under those assumptions, the tangent space to $\mathcal{M}_{t}$ at $X$ is $T_{X} \mathcal{M}_{t}=\operatorname{Ker} D q_{t}(X)$, that is,

$$
T_{X} \mathcal{M}_{t}=\left\{\delta x \in \mathbb{R}^{d} \mid D q_{t}(X) \delta x=0\right\} .
$$

Letting $e=\Pi(\hat{X}, X)$, we can define the differential of $q_{t}$ with respect to error $e$ as $D q_{t}^{e}\left(\hat{X}_{t}\right):=D q_{t}\left(\hat{X}_{t}\right)\left(D \Pi\left(\hat{X}_{t}\right)\right)^{-1}$ along the lines of $(22)$, that is

$$
q_{t}(X)-q_{t}(\hat{X})=D q_{t}^{e}(\hat{X}) e+O\left(\|e\|^{2}\right)
$$

Definition 9. The nonlinear error $e=\Pi(\hat{X}, X)$ is said compatible with the constraints $\mathcal{M}_{t}$ if, for each $t \geq 0$, there exists a $k$-dimensional linear subspace $\vec{W}_{t} \subset \mathbb{R}^{d}$ such that $\operatorname{Ker} D q_{t}^{e}(X)=\vec{W}_{t}$ for all $X \in \mathcal{M}_{t}$.

As $\mathcal{M}_{t}$ is assumed of the form $q_{t}^{-1}(c)$, this definition admits a more practical alternative formulation.

Proposition 8 (Alternative definition). If $\operatorname{Ker} D q_{t}^{e}(X)$ (viewed as a function of $X$ ) is a function of $q_{t}(X)$ only, then the nonlinear error $e$ is compatible with the constraint $q_{t}(x)=c$ in the sense of Definition 9.

This is the simple condition highlighted in Section 1.1. It is slightly more restrictive than Definition 9 since it is required to hold for all $c$, but proves more practical. 
Theorem 1. Assume the nonlinear error $e=\Pi(\hat{X}, X)$ is compatible with the constraints $\mathcal{M}_{t}$. Then, the EKF based on the nonlinear error $e$ is consistent with the constraints in the sense of Definition 7, that is, $1-\hat{X}_{t} \in \mathcal{M}_{t}$, and ${ }^{2}-\operatorname{Im} P_{t}^{e} \subset \operatorname{Ker} D q_{t}^{e}\left(\hat{X}_{t}\right), \forall t \geq 0$, if it is initalized such that 1 and 2 are satisfied at $t=0$.

Note indeed that, as $P_{t}^{e}$ reflects the dispersion of alternative error $e$, in condition 2-, we had to translate Definition 7 into conditions related to $e$.

Proof. Propagation step: Start from the initial belief at $t=0$, and consider a first phase of propagation. During this phase, the estimate $\hat{X}_{t}$ naturally remains in $\mathcal{M}_{t}$. Let us verify that during propagation $\operatorname{Im} P_{t}^{e} \subset \operatorname{Ker} D q_{t}^{e}\left(\hat{X}_{t}\right)$, i.e., $D q_{t}^{e}\left(\hat{X}_{t}\right) P_{t}^{e}=0$, or equivalently $D q_{t}^{e}\left(\hat{X}_{t}\right) P_{t}^{e} D q_{t}^{e}\left(\hat{X}_{t}\right)^{T}=0$ owing to the fact that $P_{t}^{e}$ is symmetric. Let $P_{t}$ denote the conventional EKF covariance matrix. Recall from Proposition 7 that the image of $P_{t}$ remains inside the tangent space at $\hat{X}_{t}$, that is, $D q_{t}\left(\hat{X}_{t}\right) P_{t} D q_{t}\left(\hat{X}_{t}\right)^{T}=0$. As we have $D q_{t}^{e}\left(\hat{X}_{t}\right)=D q_{t}\left(\hat{X}_{t}\right)\left(D \Pi\left(\hat{X}_{t}\right)\right)^{-1}$, see (15) and (25), it would then suffice to prove the relation

$$
D \Pi\left(\hat{X}_{t}\right) P_{t} D \Pi\left(\hat{X}_{t}\right)^{T}=P_{t}^{e}
$$

remains true if it is initially true, to readily prove $D q_{t}^{e}\left(\hat{X}_{t}\right) P_{t}^{e} D q_{t}^{e}\left(\hat{X}_{t}\right)^{T}=0$.

To prove (26) it suffices to prove that if $P_{t}^{e}$ satisfies Riccati equation $\frac{d}{d t} P_{t}^{e}=A_{t}^{e} P_{t}^{e}+$ $P_{t}^{e}\left(A_{t}^{e}\right)^{T}$, the matrix $D \Pi\left(\hat{X}_{t}\right)^{-1} P_{t}^{e}\left(D \Pi\left(\hat{X}_{t}\right)^{-1}\right)^{T}$ satisfies $\frac{d}{d t} P_{t}=A_{t} P_{t}+P_{t} A_{t}^{T}$. Denote $D \Pi\left(\hat{X}_{t}\right)^{-1}=N_{t}$. We have $\frac{d}{d t} N_{t} P_{t}^{e} N_{t}^{T}=\dot{N}_{t} P_{t}^{e} N_{t}^{T}+N_{t} \dot{P}_{t}^{e} N_{t}^{T}+N_{t} P_{t}^{e} \dot{N}_{t}^{T}=\dot{N}_{t} P_{t}^{e} N_{t}^{T}+$ $N_{t}\left(A_{t}^{e} P_{t}^{e}+P_{t}^{e}\left(A_{t}^{e}\right)^{T}\right) N_{t}^{T}+N_{t} P_{t}^{e} \dot{N}_{t}^{T}=A_{t}\left(N_{t} P_{t}^{e} N_{t}^{T}\right)+\left(N_{t} P_{t}^{e} N_{t}^{T}\right) A_{t}^{T}$, provided that the relation $A_{t}^{e}=N_{t}^{-1}\left[A_{t} N_{t}-\dot{N}_{t}\right]$ holds true. This is the case as if we let $\delta X_{t}=\hat{X}_{t}-X_{t}$, we have $\frac{d}{d t} \delta X_{t}=A_{t} \delta X_{t}, \frac{d}{d t} e_{t}=A_{t}^{e} e_{t}$, and $N_{t} e_{t}=\delta X_{t}$ up to first order terms in $\delta X_{t}$. Thus $\frac{d}{d t} N_{t}+N_{t} A_{t}^{e}=A_{t} N_{t}$, proving the result.

Update step: Just before the first update, at $t=t_{0}>0$, we have just shown that 1- $\hat{X}_{t_{0}} \in \mathcal{M}_{t_{0}}$, and 2- $\operatorname{Im} P_{t_{0}}^{e} \subset \operatorname{Ker} D q_{t_{0}}^{e}\left(\hat{X}_{t_{0}}\right)$. We first need to check that $\hat{X}_{t_{0}}^{+} \in$ $\mathcal{M}_{t_{0}}$. According to our update definition (20), (21), (23), we have $\hat{X}_{t_{0}}^{+}=\tilde{\chi}(1)$ where $\hat{X}_{t_{0}}=\tilde{\chi}(0)$ and $\tilde{\chi}(s)$ satisfies $\frac{d}{d s} \tilde{\chi}(s)=D \Pi(\tilde{\chi}(s))^{-1} K_{0} z_{0}$. Besides, we have $K_{0} z_{0} \in$ $\operatorname{Im} P_{t_{0}}^{e} \subset \operatorname{Ker} D q_{t_{0}}^{e}\left(\hat{X}_{t_{0}}\right)$, owing to the definition of the gain $K_{0}$. Due to our compatibility assumption of Definition 9 that the Kernel of $D q_{t_{0}}^{e}$ is fixed over $\mathcal{M}_{t}$, we have $\operatorname{Ker} D q_{t_{0}}^{e}\left(\hat{X}_{t_{0}}\right)=\operatorname{Ker} D q_{t_{0}}^{e}(X):=\operatorname{Ker} D q_{t_{0}}(X) D \Pi(X)^{-1}$ for all $X \in \mathcal{M}_{t_{0}}$, proving that $D \Pi(X)^{-1} K_{0} z_{0} \in T_{X} \mathcal{M}_{t_{0}}$, as $D q_{t_{0}}$ was required to have constant rank. Thus the vector field $\left\{D \Pi(X)^{-1} K_{0} z_{0}, X \in \mathcal{M}_{t_{0}}\right\}$ is tangent to $\mathcal{M}_{t_{0}}$. By unicity of solutions of differential equations, the solution of $\frac{d}{d s} \tilde{\chi}(s)=D \Pi(\tilde{\chi}(s))^{-1} K_{0} z_{0}$ is thus a curve contained in $\mathcal{M}_{t_{0}}$, and in particular $\tilde{\chi}(1)=\hat{X}_{t_{0}}^{+} \in \mathcal{M}_{t_{0}}$.

Regarding condition 2-, as we have proved that $\operatorname{Im} P_{t_{0}}^{e} \subset \operatorname{Ker} D q_{t_{0}}^{e}\left(\hat{X}_{t_{0}}\right)$, we immediately see from equations (19), (21) that $\operatorname{Im}\left(P_{t_{0}}^{e}\right)^{+} \subset \operatorname{Im} P_{t_{0}}^{e} \subset \operatorname{Ker} D q_{t_{0}}^{e}\left(\hat{X}_{t_{0}}\right)$. Under our compatibility assumption of Definition 9 , and since we have just proved that $\hat{X}_{t_{0}}^{+} \in \mathcal{M}_{t_{0}}$, we have $\operatorname{Ker} D q_{t_{0}}^{e}\left(\hat{X}_{t_{0}}\right)=\operatorname{Ker} D q_{t_{0}}^{e}\left(\hat{X}_{t_{0}}^{+}\right)$, which implies indeed $\operatorname{Im}\left(P_{t_{0}}^{e}\right)^{+} \subset \operatorname{Ker} D q_{t_{0}}^{e}\left(\hat{X}_{t_{0}}^{+}\right)$.

The extension to arbitrary $t \geq t_{0}$ is easily done by induction, as we have proved both propagation and update steps preserve the two conditions we pursue. 


\subsection{Complementary results in the presence of process noise}

The results may be extended beyond systems with deterministic dynamics and noisy measurements, sometimes referred to as output error systems [22]. Indeed, consider dynamics (1), and assume it is corrupted by noise as follows (Stratonovitch's stochastic integral) :

$$
\frac{d}{d t} X_{t}=f\left(X_{t}, u_{t}\right)+\bar{G}\left(X_{t}\right) w_{t}
$$

where $w_{t}$ is a Langevin noise with covariance $Q_{t}$. Then, an EKF based on error $e_{t}$ can also be devised but it must take into account the dispersion of the error under the noise. The Riccati equation for system (27) of the conventional EKF writes $\frac{d}{d t} P_{t}=$ $A_{t} P_{t}+P_{t} A_{t}^{T}+\bar{G}_{t} Q_{t} \bar{G}_{t}^{T}$, with $\bar{G}_{t}:=\bar{G}\left(\hat{X}_{t}\right)$. Recalling (15), the conventional dispersion term $\bar{G}_{t} Q_{t} \bar{G}_{t}^{T}$ associated to error $\delta X$, naturally writes $D \Pi\left(\hat{X}_{t}\right) \bar{G}_{t} Q_{t} \bar{G}_{t}^{T} D \Pi\left(\hat{X}_{t}\right)^{T}$ in terms of error $e_{t}$.

Thus, an EKF based on error $e_{t}$ for system (27) can be defined as in Section 4.1, with the only difference that covariance propagates as

$$
\frac{d}{d t} P_{t}^{e}=A_{t}^{e} P_{t}^{e}+P_{t}^{e}\left(A_{t}^{e}\right)^{T}+D \Pi\left(\hat{X}_{t}\right) \bar{G}_{t} Q_{t} \bar{G}_{t}^{T} D \Pi\left(\hat{X}_{t}\right)^{T} .
$$

Proposition 9. Consider noisy dynamics (27), and assume the noise diffuses inside $\mathcal{M}_{t}$, i.e., $\operatorname{Im}\left(\bar{G}\left(X_{t}\right)\right) \in T_{X_{t}} \mathcal{M}_{t}$ for any $t>0$. Then Theorem 1 still holds true.

Proof. We have to prove $D q_{t}^{e}\left(\hat{X}_{t}\right) P_{t}^{e}=0$ for any $t>0$. Differentiating the relation gives: $\frac{d}{d t}\left(D q_{t}^{e}\left(\hat{X}_{t}\right) P_{t}\right)=\left(\frac{d}{d t} D q_{t}^{e}\left(\hat{X}_{t}\right)\right) P_{t}^{e}+D q_{t}^{e}\left(\hat{X}_{t}\right)\left(A_{t} P_{t}+P_{t} A_{t}^{T}+D \Pi\left(\hat{X}_{t}\right) \bar{G}_{t} Q_{t} \bar{G}_{t}^{T} D \Pi\left(\hat{X}_{t}\right)^{T}\right)$. By definition $D q_{t}^{e}\left(\hat{X}_{t}\right) D \Pi\left(\hat{X}_{t}\right) \bar{G}_{t}=D q_{t}\left(\hat{X}_{t}\right) \bar{G}_{t}$, which is equal to 0 since we assumed $\operatorname{Im}\left(\bar{G}\left(\hat{X}_{t}\right)\right) \in T_{\hat{X}_{t}} \mathcal{M}_{t}$. Thus $\frac{d}{d t}\left(D q_{t}^{e}\left(\hat{X}_{t}\right) P_{t}\right)=\left(\frac{d}{d t} D q_{t}^{e}\left(\hat{X}_{t}\right)\right) P_{t}^{e}+D q_{t}^{e}\left(\hat{X}_{t}\right)\left(A_{t} P_{t}+P_{t} A_{t}^{T}\right)$, which is the same as in the noise free case. By unicity of the solution $D q_{t}^{e}\left(\hat{X}_{t}\right) P_{t}^{e}=$ $0, \forall t>0$.

\section{$5 \quad$ The Lie group case}

Coming up with a nonlinear error that is compatible with all the constraints $\mathcal{M}_{t}$ may prove difficult in practice. However, this can be done automatically in the case where the state space is a Lie group, under a set of conditions.

\subsection{A brief primer on Lie groups}

For a complete introduction to Lie groups, the reader is referred to [25]. A $d$-dimensional Lie group $G$ is a smooth manifold endowed with a group structure, such that the group operation and the inverse map are smooth. In this paper, we will only consider matrix Lie groups, that is, we will assume that $G \subset \mathbb{R}^{N \times N}$ is a subgroup of the space of invertible square matrices. All the results are valid for a general Lie group, but matrix Lie groups provide a more concrete picture.

The tangent space $T_{I} G$ at the identity matrix $I$, is called the Lie algebra of $G$, and is denoted by $\mathfrak{g} \subset \mathbb{R}^{N \times N}$. It has dimension $d$, as the group. In turn, $\mathfrak{g}$ may be identified with the vector space $\mathbb{R}^{d}$, through a map $\mathbb{R}^{d} \rightarrow \mathfrak{g}$ that we denote $\xi \mapsto \xi^{\wedge}$. There is a natural 
map between $\mathfrak{g}$ and $G$, called the exponential map of $G$, and denoted by exp, which is

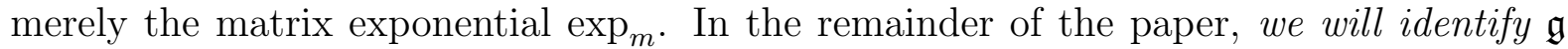
to $\mathbb{R}^{d}$, and we will directly denote $\exp (\xi)=\exp _{m}\left(\xi^{\wedge}\right)$ the exponential of $\xi^{\wedge} \in \mathfrak{g}$. The exponential defines a local diffeomorphism between $\mathfrak{g}$ and an open subset of $G$ containing the identity. Its local inverse is called the Lie logarithm. In general, the exponential is not surjective.

Recalling our identifying $\mathfrak{g}$ with $\mathbb{R}^{d}$ we define the Lie bracket of $\xi, \zeta \in \mathbb{R}^{d}$ as $[\xi, \zeta]:=$ $\left[\xi^{\wedge}, \zeta^{\wedge}\right]_{\mathfrak{g}}$ where $[\cdot, \cdot]_{\mathfrak{g}}$ denotes the Lie bracket in $\mathfrak{g}$, and call Lie subalgebra $L$ any linear subspace of $\mathbb{R}^{d}$ such that $[\xi, \zeta] \in L$ for $\xi, \zeta \in L$. The Baker-Campbell-Hausdorff (BCH) formula states $\exp (\xi) \exp (\zeta)=\exp (\eta)$ with $\eta=\xi+\zeta+$ Lie bracket terms composed from $\xi$ and $\zeta$, see $[25]$.

Similarly, the tangent space $T_{g} G$ at arbitrary $g \in G$ can be identified to $\mathbb{R}^{d}$ through left and right multiplications, i.e., the matrices $g \xi^{\wedge}$ and $\left(\xi^{\wedge}\right) g$ are both (distinct) vectors of $T_{g} G$. Throughout this section, we will privilege left multiplications, for exposition purposes. To linearize a map $h: G \mapsto \mathbb{R}^{p}$, at an arbitrary point $g \in G$, we can evaluate how it changes by infinitesimally following an arbitrary tangent vector $g \xi^{\wedge}$ at $g$. The left linear approximation to $h: G \rightarrow \mathbb{R}^{p}$ at $g$ in the direction $\xi \in \mathbb{R}^{d}$ can then be defined as the matrix $H \in \mathbb{R}^{p \times d}$ such that $h(g \exp (\xi))-h(g)=H \xi+O\left(\|\xi\|^{2}\right)$, that is, $H \xi=\left.\frac{d}{d s} h(g \exp (s \xi))\right|_{s=0}$. One must then bear in mind that infinitesimal shifts at any $g \in G$ are thus always represented by elements of $\mathbb{R}^{d}$.

\subsection{Group affine systems on Lie groups}

Consider deterministic dynamics on a Lie group $G$ with state $\chi_{t} \in G$ defined by :

$$
\frac{d}{d t} \chi_{t}=f\left(\chi_{t}, u_{t}\right):=f_{u_{t}}\left(\chi_{t}\right)
$$

Definition 10. For two trajectories $\chi_{t}$ and $\bar{\chi}_{t}$ of the system (28), the left-invariant error $\eta_{t}$ is defined as:

$$
\eta_{t}=\chi_{t}^{-1} \bar{\chi}_{t}
$$

The right-invariant error between two trajectories is $\eta_{t}=\chi_{t} \bar{\chi}_{t}{ }^{-1}$. A key result of [3] is as follows.

Theorem 2 ( [3]). For the dynamics (28), the left- and right-invariant errors satisfy an equation of the form

$$
\frac{d}{d t} \eta_{t}=g_{u_{t}}\left(\eta_{t}\right)
$$

for some map $g_{u_{t}}=G \mapsto T_{\eta_{t}} G$, if and only if

$$
\forall a, b \in G, f_{u_{t}}(a b)=a f_{u_{t}}(b)+f_{u_{t}}(a) b-a f_{u_{t}}(I) b .
$$

In that case, the dynamics are called "group-affine", and errors are (slightly abusively) said to have an autonomous evolution (30), in the sense it does not explicitly depend on $\chi_{t}$. Moreover, we have

$$
\forall a, b, g_{u_{t}}(a b)=a g_{u_{t}}(b)+g_{u_{t}}(a) b \text {. }
$$


The group-affine property (31) encompasses many systems of engineering interest revolving around attitude estimation, localization, and SLAM, see [1,3,4], and notably includes invariant systems.

Another key result of [3] is the fact the error system is encoded by a linear differential equation, see Corollary 1 . This stems from the fact that $g_{u_{t}}$ necessarily satisfies (32). The theory is concisely recapped as follows.

Definition 11. Group automorphisms are the maps $\Phi: G \rightarrow G$ such that $\forall a, a^{\prime} \in$ $G, \Phi\left(a a^{\prime}\right)=\Phi(a) \Phi\left(a^{\prime}\right)$. The Lie algebra automorphisms are the linear maps $\Psi$ such that $\forall \xi, \zeta$ we have $\Psi([\xi, \zeta])=[\Psi(\xi), \Psi(\zeta)]$.

A very well-known and prototypical automorphism is the Adjoint operator.

Definition 12. The Adjoint operator $A d_{g}$, with $g \in G$, is a Lie algebra automorphism, defined through the equality $g \exp (\xi) g^{-1}=\exp \left(A d_{g}(\xi)\right)$.

Proposition 10. [3] Let $\Phi_{t}^{g}$ be the flow associated to the system (30). Because of (32), $\Phi_{t}^{g}$ necessarily defines an automorphism of the Lie group $G$ for all $t$.

This is because for $\eta_{0}, \eta_{0}^{\prime} \in G, \Phi_{t}^{g}\left(\eta_{0}\right) \Phi_{t}^{g}\left(\eta_{0}^{\prime}\right)$ is a solution of (30) with initial condition $\eta_{0} \eta_{0}^{\prime}$, using (32).

According to the Lie group - Lie algebra correspondence, and to the fact that $\Phi_{t}^{g}$ is an automorphism, we have necessarily:

Theorem 3. Let $d\left(g_{u_{t}}\right)_{I}: T_{I} G \rightarrow T_{I} G$ denote the differential at I of $g_{u_{t}}$ of (30). As $\mathfrak{g}=T_{I} G$ was identified to $\mathbb{R}^{d}, d\left(g_{u_{t}}\right)_{I}$ may be encoded by a matrix $A_{t} \in \mathbb{R}^{d \times d}$. Let $\Phi_{t}^{g}$ and $\Psi_{t}^{g}$ denote the flows of $\frac{d}{d t} \eta_{t}=g_{u_{t}}\left(\eta_{t}\right)$ and $\frac{d}{d t} \zeta_{t}=A_{t} \zeta_{t}$ respectively. We have:

- $\Psi_{t}^{g}$ is a Lie algebra automorphism,

- $\forall t, \forall \zeta_{0} \in \mathbb{R}^{d}, \quad \Phi_{t}^{g}\left(\exp \zeta_{0}\right)=\exp \left(\Psi_{t}^{g}\left(\zeta_{0}\right)\right)$.

This readily proves:

Corollary 1. [3] We have for all $\xi \in \mathbb{R}^{d}: \Phi_{t}^{g}(\exp \xi)=\exp \left(F_{t} \xi\right)$, where $F_{t}$ is the linear operator being solution of the operator equation $F_{0}=I, \frac{d}{d t} F_{t}=d\left(g_{u_{t}}\right)_{I} \circ F_{t}$.

\subsection{Considered problem and support of the posterior}

Consider a system defined by the noise-free dynamics (28), with the group-affine condition (31) and initial condition $\chi_{0} \in G$. Moreover, consider noisy observations $Y_{n} \in \mathbb{R}^{p}$ :

$$
Y_{n}=h\left(\chi_{t_{n}}\right)+V_{n}
$$

where the $V_{n}$ 's are Gaussian independent noises. Suppose the prior is initially supported by the subset

$$
\mathcal{M}_{0}=\chi_{0} G_{0}
$$

with $\chi_{0} \in G$, and $G_{0}$ a closed Lie subgroup of dimension $k$. Let $L_{0}$ denote its Lie algbera. $L_{0}$ is a Lie subalgebra of the Lie algebra $\mathfrak{g}$. The problem fits into the framework of Section 
3, see Remark 1. In the particular case where $G=\mathbb{R}^{d}$ endowed with addition, note (34) boils down to a $k$-dimensional affine subspace.

We can use the Lie group machinery to prove that the support of the posterior distribution not only is a manifold, but has a group structure.

Proposition 11. Let $\mathcal{M}_{t}$ denote the support of the posterior, that is, the image of $\mathcal{M}_{0}$ of (34) through the flow $\Phi_{t}^{f}$ of (28). Let $\Phi_{t}^{g}$ be the flow of (30). Then $\mathcal{M}_{t}=\chi_{t} G_{t}$, with $\chi_{t}:=\Phi_{t}^{f}\left(\chi_{0}\right)$ and $G_{t}:=\Phi_{t}^{g}\left(G_{0}\right)$. $G_{t}$ is a (closed) embedded subgroup of $G$ and its Lie algebra is $L_{t}=\Psi_{t}^{g}\left(L_{0}\right)$, where $\Psi_{t}^{g}$ is defined in Theorem 3.

Proof. By definition $\chi_{t}$ satisfies (28). Let $\tilde{\chi}_{t}$ satisfy (28). Then $\eta_{t}:=\chi_{t}^{-1} \tilde{\chi}_{t}$ satisfies (30). If $\tilde{\chi}_{0} \in \mathcal{M}_{0}$ then $\eta_{0}:=\chi_{0}^{-1} \tilde{\chi}_{0} \in G_{0}$ and as $\tilde{\chi}_{t}=\chi_{t} \eta_{t}$ we see that necessarily $\mathcal{M}_{t}=\chi_{t} G_{t}$ with $G_{t}=\Phi_{t}^{g}\left(G_{0}\right)$. The flow has continuous inverse so $G_{t}$ is closed. According to Proposition 10, $\Phi_{t}^{g}$ is a group automorphism and $\Psi_{t}^{g}$ a Lie-algebra automorphism from Theorem 3. Thus $G_{t}$ is a subgroup of $G$ and $L_{t}$ a Lie subalgebra of $\mathfrak{g}$.

\subsection{Support of the belief of EKFs based on invariant errors}

In this paragraph, we consider the EKF based on the nonlinear left-invariant error

$$
e_{t}=\Pi\left(\hat{\chi}_{t}, \chi_{t}\right):=\hat{\chi}_{t}^{-1} \chi_{t}
$$

by applying the methodology of Section 4 . The error is said left-invariant as $\Pi\left(\hat{\chi}_{t}, \chi_{t}\right)=$ $\Pi\left(g \hat{\chi}_{t}, g \chi_{t}\right) \forall g \in G$.

Remark 5. In Section 4 the state space is $\mathbb{R}^{d}$ to make the exposition more tutorial. However, the methodology readily carries over to the case where the state space is a manifold, and in particular a matrix Lie group. When referring to the methodology of Section 4, we will in fact systematically refer to its immediate extension to the Lie group case, that leverages the Lie algebra to encode tangent vectors, see Section 5.1.

Recalling Section 5.1, this immediate extension is as follows. First, we will need to linearize error (35). On $G$ this is done writing $\Pi(\hat{\chi}, \hat{\chi} \exp (\delta e))=\hat{\chi}^{-1} \hat{\chi} \exp (\delta e)=$ $\exp (\delta e)=\exp _{m}\left((\delta e)^{\wedge}\right)=I+(\delta e)^{\wedge}+O\left(\|\delta e\|^{2}\right)$, and null error corresponds to $e=I$. As a result we will refer to the linearized error as $\delta e \in \mathbb{R}^{d}$ such that $e=I+(\delta e)^{\wedge}+O\left(\|\delta e\|^{2}\right)$. Then, we will need to define the differential $H^{e}$ of any map $h: G \rightarrow \mathbb{R}^{p}$ with respect to error $e$. To do so, we proceed as in Section 4 , and for any $e \in G$, we let $h(\chi)-h(\hat{\chi})=$ $H^{e} \delta e+O\left(\|\delta e\|^{2}\right)$, where $\Pi(\hat{\chi}, \chi)=e=\exp (\delta e)$, that is, where we let $\chi=\hat{\chi} \exp \delta e$.

The following theorem is our second major result.

Theorem 4. Consider system (28) with the group-affine condition (31), with observations (33), and let $\mathcal{M}_{t}$ be the image of $\mathcal{M}_{0}$ of (34) through the flow $\Phi_{t}^{f}$ of (28). The EKF based on the left-invariant error $e_{t}$ of (35) is consistent with the constraints $\mathcal{M}_{t}$ in the sense of Definition 7 . In other terms, all the estimates $\hat{\chi}_{t}$ are elements of $\mathcal{M}_{t}$ at all times, and the covariance matrix $P_{t}^{e}$ indicates dispersion only whithin the tangent space to $\mathcal{M}_{t}$ at $\hat{\chi}_{t}$, and null dispersion transversally to it.

Moreover, exactly the same holds if the EKF is based on the right-invariant error $e_{t}=\Pi\left(\hat{\chi}_{t}, \chi_{t}\right)=\chi_{t} \hat{\chi}_{t}^{-1}$. 
Proof. This stems from the theory of Section 4.3. To apply Theorem 1, we only need to prove $e_{t}$ is compatible with the constraints $\mathcal{M}_{t}$, i.e. the condition of Proposition 8 . To do so we use the quotient manifold theorem 7.15 of [20], that states that if $G_{t}$ is a closed subgroup of $G$, then the quotient map $q_{t}: G \rightarrow G / G_{t}$ associated with the left coset space $G / G_{t}$ is a smooth submersion. Let $\hat{\chi} \in \mathcal{M}_{t}:=\chi_{t} G_{t}$. For left-invariant errors (35), we proved linearized errors satisfy $(\delta e)^{\wedge}=\left.\frac{d}{d s}\right|_{s=0} \Pi(\hat{\chi}, \hat{\chi} \exp (s \delta e))$. Thus $\operatorname{Ker} D q_{t}^{e}(\hat{\chi})$ consists of elements $\delta e \in T_{I} G$ such that $\left.\frac{d}{d s}\right|_{s=0} \hat{\chi} \exp (s \delta e) \in T_{\hat{\chi}} \mathcal{M}_{t}$. This means Ker $D q_{t}^{e}(\hat{\chi})=\left\{\xi \in \mathbb{R}^{d} \mid \hat{\chi} \xi^{\wedge} \in T_{\hat{\chi}} \mathcal{M}_{t}\right\}=\left\{\xi \in \mathbb{R}^{d} \mid \chi_{t}^{-1} \hat{\chi} \xi^{\wedge} \in T_{\chi_{t}^{-1} \hat{\chi}} G_{t}\right\}$. Using left translation by $\chi_{t}^{-1} \hat{\chi}$ we see the condition is equivalent to $\xi$ being in the Lie algebra of $G_{t}$, which is $L_{t}$. And thus $\operatorname{Ker} D q_{t}^{e}(\hat{\chi})=T_{\hat{\chi}} \mathcal{M}_{t}=L_{t}$. As $L_{t}=\psi_{t}^{g}\left(L_{0}\right)$ is independent of chosen $\hat{\chi} \in \mathcal{M}_{t}$, the condition of Proposition 8 is trivially met. Regarding right-invariant errors, (34) may be re-written $\mathcal{M}_{0}=\exp \left(L_{0}\right) \chi_{0}=\chi_{0} \exp \left(L_{0}^{\prime}\right)$ with $L_{0}^{\prime}=A d_{\chi_{0}}^{-1}\left(L_{0}\right)$ which is a subalgebra. Switching left and right the proof is identical.

Remark 6. The result is remarkable, as it shows that a single EKF achieves consistency of the belief support for a very large class of (time-varying) constraints all at once, and without ad-hoc adaptation. Indeed, it suffices that $\mathcal{M}_{0}=\chi_{0} G_{0}$ with $G_{0}$ a subgroup of dimension $k \leq d$ for the implied constraints on the support to be met at all times. This in sharp contrast with the usual state constrained solutions [23], where each constraint generates an ad-hoc adaptation of the EKF's output estimate. Note that, the EKF may be based on left or right-invariant error, leading to two different filters that both are consistent with the constraints.

\subsection{Explicit derivation of EKFs based on invariant estimation errors}

We have not actually derived the equations for the EKF based on error $e_{t}$ of (35). It turns out that 1- the map $\varphi$ of Definition 8 is merely the Lie exponential (hence its name), and 2- for specific classes of output maps, we recover the invariant EKF (IEKF) of [3].

Lemma 1. Consider the map $\varphi$ of Definition 8 for the left-invariant error $e_{t}$ of (35). Then for $\hat{\chi} \in G$ and $\delta e \in \mathbb{R}^{d}$, it simply writes

$$
\varphi(\hat{\chi}, \delta e)=\hat{\chi} \exp (\delta e)
$$

For a right-invariant error $e_{t}=\chi_{t} \hat{\chi}_{t}^{-1}$ we have instead

$$
\varphi(\hat{\chi}, \delta e)=\exp (\delta e) \hat{\chi}
$$

Proof. The Lie exponential of $\delta e \in \mathbb{R}^{d}$ gets its name from the fact that it satisfies $\exp (\delta e)=\tilde{\chi}(1) \in G$, with $\tilde{\chi}(0)=I$ and where $\tilde{\chi}$ satisfies the differential equation $\frac{d}{d s} \tilde{\chi}(s)=\tilde{\chi}(s) \delta e \in T_{\tilde{\chi}(s)} G$.

On the other hand, we can apply Definition 8. The latter definition uses coordinates in $\mathbb{R}^{d}$, and needs to be defined more abstractly to match the fact the state space is a group, that is, a curved space, see Remark 5 . This is immediate, though, since $\varphi(\hat{\chi}, \delta e)$ of Definition 8 is in fact defined as $\tilde{\chi}(1)$ where $\tilde{\chi}(0)=\hat{\chi}$, and where $\frac{d}{d s} \tilde{\chi}(s) \in T_{\tilde{\chi}(s)} G$ is a tangent vector $\tilde{\chi}(s) \xi(s)^{\wedge}$ at $\tilde{\chi}(s)$ that corresponds to infinitesimally shifting $\tilde{\chi}(s)$ in direction of error $\delta e$, i.e., such that $\Pi(\tilde{\chi}(s), \tilde{\chi}(s) \exp (\xi(s)))=I+\delta e^{\wedge}+O\left(\|\xi\|^{2}\right)$. As a result $\xi(s) \equiv \delta e$. Thus $\varphi(\hat{\chi}, \delta e)=\tilde{\chi}(1)=\hat{\chi} \exp (\delta e)$. 
The full correspondance with IEKF is as follows:

Theorem 5. For the system (28) observed through noisy measurements (33) where we let $h\left(\chi_{t_{n}}\right)=\chi_{t_{n}} \cdot \bar{d}$ for some $\bar{d} \in \mathbb{R}^{N}$, the EKF based on the left-invariant error (35) along the lines of Section 4 actually is the left invariant EKF (LIEKF) of [3].

When on the other hand $h\left(\chi_{t_{n}}\right):=\chi_{t_{n}}^{-1} \cdot \bar{d} \in \mathbb{R}^{N}$, then the EKF based on the rightinvariant error $\Pi\left(\hat{\chi}_{t}, \chi_{t}\right)=\chi_{t} \hat{\chi}_{t}^{-1}$ is the RIEKF of [3].

The proof has been moved to the Appendix.

\subsection{A theoretical example}

Our preliminary conference paper [12] was devoted to systems of the form $\frac{d}{d t} \chi_{t}=\chi_{t} u_{t}+$ $\nu_{t} \chi_{t}$ with $\chi_{t}$ the state belonging to a Lie group $G$, with measurements of the form $Y_{n}=$ $\chi_{t_{n}} \cdot \bar{d}+V_{n}$. It was proved that for the latter systems, the IEKF is compatible with constraints of the form $\chi_{t} \cdot b_{t}=c_{t}$ where of course $b_{t}, c_{t} \in \mathbb{R}^{N}$ are such that the latter equality is preserved by (28). To prove this is a direct consequence of the theory of Section 5, all we need to show is that 1 - systems of the form $\frac{d}{d t} \chi_{t}=\chi_{t} u_{t}+\nu_{t} \chi_{t}$ are group-affine, and 2- the set $\mathcal{M}_{t}=\left\{\chi \in G \mid \chi \cdot b_{t}=c_{t}\right\}$ is of the form $\mathcal{M}_{t}=\bar{\chi}_{t} G_{t}$, with $G_{t}$ a closed subgroup. The first point is easily checked, and has already been noticed in [3]. Regarding the second, we have $\mathcal{M}_{t}=\bar{\chi}_{t} G_{t}$ with $\bar{\chi}_{t}$ any element such that $\bar{\chi}_{t} b_{t}=c_{t}$, and $G_{t}=\left\{\tilde{\chi} \in G \mid \tilde{\chi} b_{t}=b_{t}\right\}$. Note that, this shows in passing that the theory of the present section is more general than [12].

\section{Applications}

\subsection{Navigation example}

In the example of Figure 1, the unicycle model serves as simplified dynamical equations $\frac{d}{d t} \theta_{t}=\omega_{t}, \frac{d}{d t} x_{t}^{(1)}=u_{t} \cos \theta_{t}, \frac{d}{d t} x_{t}^{(2)}=u_{t} \sin \theta_{t}$, where the state $\left(\theta_{t}, x_{t}\right)$ consists of the orientation $\theta_{t} \in \mathbb{S}^{1}$ of the car in the plane, and its position $x_{t}=\left(x_{t}^{(1)}, x_{t}^{(2)}\right)^{T} \in \mathbb{R}^{2}$. The angular and linear velocities $\omega_{t}, u_{t} \in \mathbb{R}$ are computed from the wheel speed sensors. As they are assumed to be perfect, the dynamics are noise free. Assume that, at discrete times $0<t_{1}<t_{2}, \cdots$ a GPS-like sensor returns the noisy position of the car, that is, measurements of the form $Y_{n}=\left(x_{t_{n}}^{(1)}, x_{t_{n}}^{(2)}\right)+V_{n}$ where $V_{n}$ is a random variable. Suppose that initially the absolute position of the car is perfectly known, that is, $x_{0}^{(1)}=x_{0}^{(2)}=0$, and that the orientation is uncertain, say, $\operatorname{Var}\left(\theta_{0}\right)=\pi / 2$ and $\operatorname{Var}\left(x_{0}^{(1)}\right)=\operatorname{Var}\left(x_{0}^{(2)}\right)=0$.

As shown in [2], the information that we have at time $t$ is of the form $q\left(\theta_{t}, x_{t}\right)=b_{t}$ with $q(\theta, x)=R(\theta)^{T} x$ with $R(\theta)$ the planar rotation of angle $\theta$. Moreover, a nonlinear error can be defined as $e_{t}=\left(e_{\theta}, e_{x}\right):=\left(\hat{\theta}_{t}-\theta_{t}, R\left(\theta_{t}\right)^{T}\left(\hat{x}_{t}-x_{t}\right)\right)$. Then, $q\left(\theta_{t}, x_{t}\right)-$ $q\left(\hat{\theta}_{t}, \hat{x}_{t}\right)=R\left(\theta_{t}\right)^{T} x_{t}-R\left(\hat{\theta}_{t}\right)^{T} \hat{x}_{t}=-e_{x}+e_{\theta} J R\left(\hat{\theta}_{t}\right)^{T} \hat{x}_{t}+O\left(\left\|e_{t}\right\|^{2}\right)$, where $J$ is the skewsymmetric matrix $[0,1 ;-1,0]$. As $q(\theta, x)=R(\theta)^{T} x$, we have $\left.D q^{e}\right|_{\hat{\theta}, \hat{x}}\left(e_{\theta}, e_{x}\right)=-e_{x}+$ $e_{\theta} J q(\hat{\theta}, \hat{x})$, so it depends on the state only via $q(\hat{\theta}, \hat{x})$, so the error is compatible, see Proposition 8, and Theorem 1 applies.

Thus, the main result of [2],which is that the updates of the EKF based on $e$ (which coincides with the IEKF, see Section 5.5) always move the car along the appropriate 


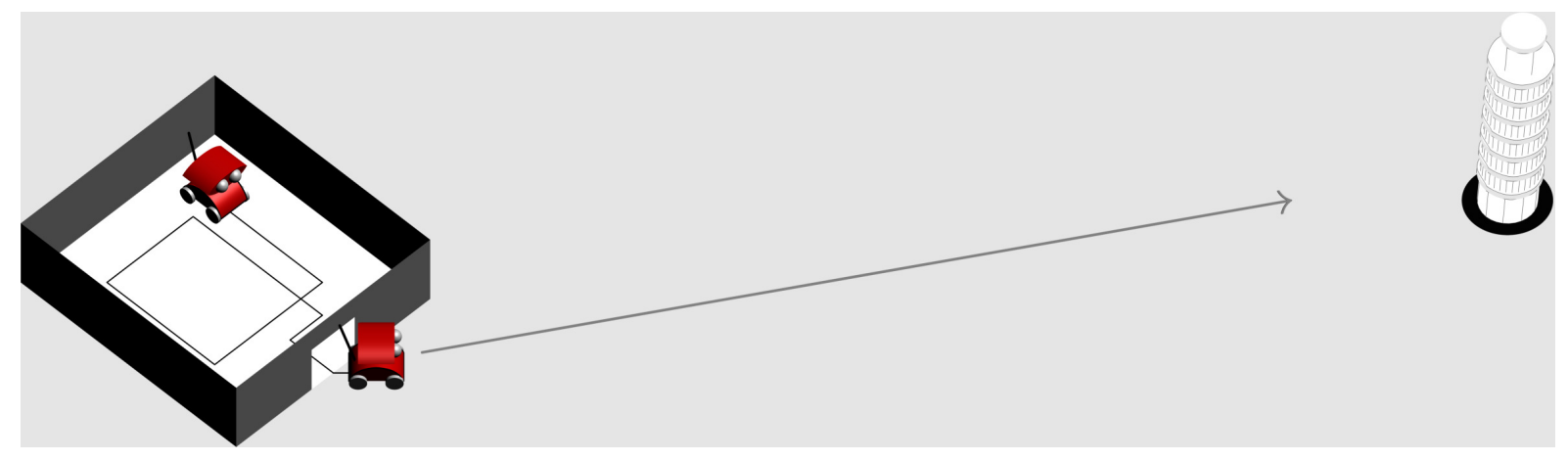

Figure 3: Numerical experiment. A robot having approximately known initial position and unknown orientation explores a room, and builds a map of 8 characteristic unknown features. Then, it leaves the building until it observes the bearing of a clearly visible landmark with known location (the Pisa tower). This new observation brings information about its orientation, and consequently on the general orientation of the map it has built.

circle, see Figure 1, with belief tangential to it, now appears as a consequence of the proposed general theory. Moreover, the fact the IEKF is compatible with the constraints was key to prove global convergence properties of the IEKF for the present problem in [2], whereas simulations show the standard EKF does actually not converge, even for small initial errors.

Remark 7. The constraint set $\mathcal{M}_{t}$ varies with time. Moreover, even for fixed $t$, each particular motion $\left(\omega_{s}, u_{s}\right)_{0 \leq s \leq t}$ of the vehicle generates a different set $\mathcal{M}_{t}$. Yet, the EKF based on e automatically meets all the constraints at all times, regardless of the motion, and $\mathcal{M}_{t}$ never needs to be computed. This is in contrast with standard EKF state constrained techniques.

\subsection{Simultaneous localization and mapping (SLAM) with par- tial prior map}

\subsubsection{Problem and results}

The following example revolves around the field of SLAM, which has drawn a lot of attention over the past two decades in the robotics community, see e.g. [14]. A wheeled robot equipped with a Red Green Blue depth (RGBD) sensor (such as the Kinect) navigates in an unknown building, and builds a map that consists of characteristic static features that the robot can track through its vision system. In the present example we assume the robot moves in a $2 \mathrm{D}$ plane and is modeled by the unicycle equations as in Section 6.1 , see also [14,27]. The dynamics of the SLAM problem thus write:

$$
\begin{aligned}
\frac{d}{d t} \theta_{t} & =\omega_{t}, \quad \frac{d}{d t} x_{t}=R\left(\theta_{t}\right)\left(u_{t}, 0\right)^{T}, \\
\frac{d}{d t} p_{t}^{i} & =0 \text { for } 1 \leqslant i \leqslant k,
\end{aligned}
$$

where the state consists of the robot's position $x_{t}=\left(x_{t}^{(1)}, x_{t}^{(2)}\right)^{T} \in \mathbb{R}^{2}$, its orientation $\theta_{t} \in \mathbb{S}^{1}$, and the positions $p^{1}, p^{2}, \ldots, p^{m} \in \mathbb{R}^{2}$ of $m$ unknown static features, and where 

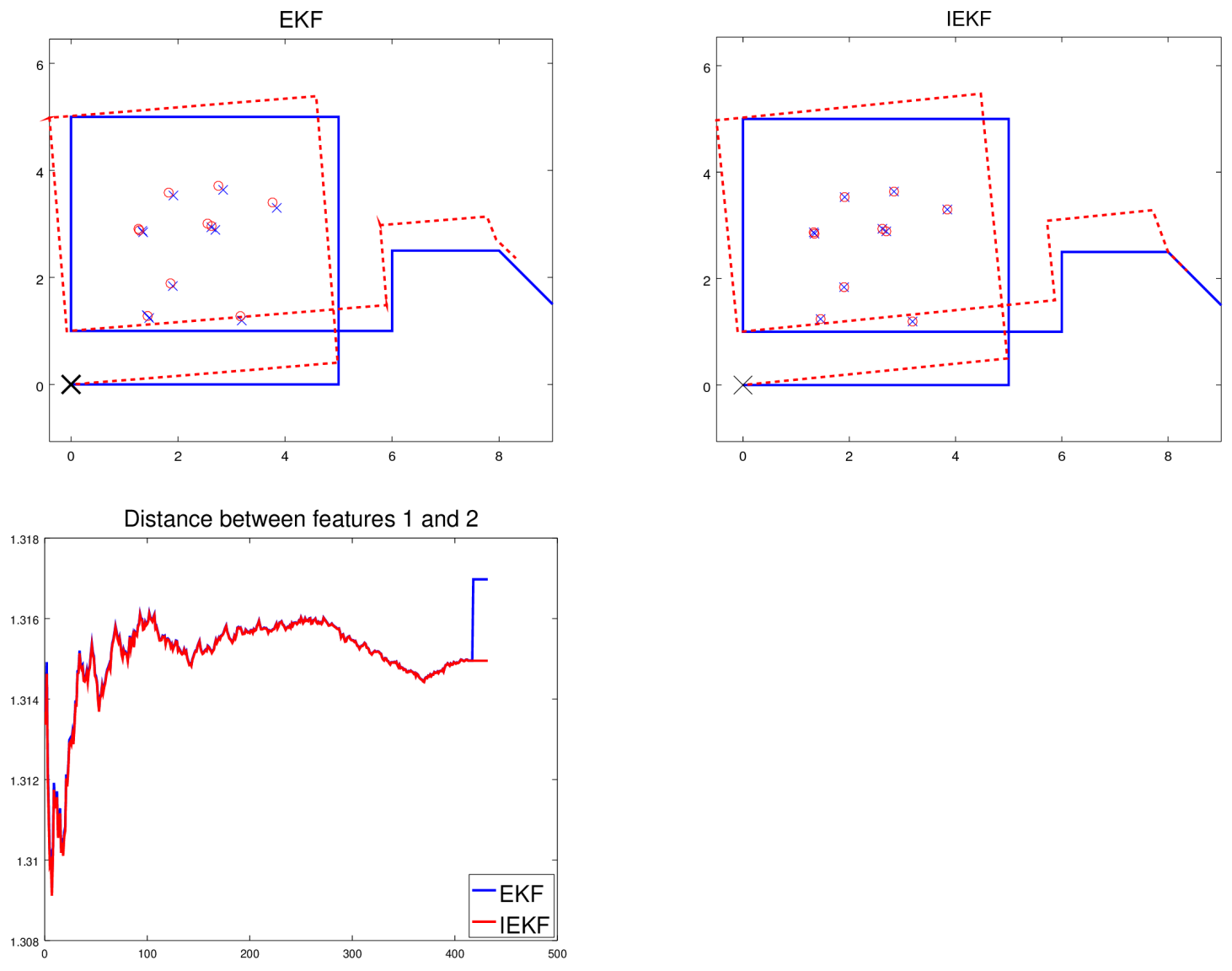

Figure 4: Left and center plots: estimations of EKF and EKF with an adapted error $e$ (dashed), true trajectory (plain), true feature locations (crosses) and estimated feature locations (circles). During the indoors phase, the robot builds a very acurate map up to a global rotation and translation. Thus the filters ends up with a covariance matrix very close to being singular: only the heading is still to be observed but the general shape of the map is known. In particular, the distances between features should not change anymore even if information regarding global position and heading is then obtained. Right plot (best seen in color) shows that they do for the EKF, meaning the shape of the map is changed by the observation, whereas it remains steady for the IEKF despite seeing the Pisa tower at time step 417. This is the point of the present paper: an information acquired by an EKF-like algorithm can be maintained over time only as long as the chosen state error variable of the filter displays "compatibility" with this information. 
the measurements $\omega_{t}, u_{t} \in \mathbb{R}$ are obtained using differential wheel speeds. Observations are made at discrete times $0<t_{1}<t_{2}, \cdots$, and consist of the positions of the features viewed from the robot $Y_{n}^{i}=R\left(\theta_{t_{n}}\right)^{T}\left(p^{i}-x_{t_{n}}\right)+V_{n}^{p}, 1 \leq i \leq m$ when those features are sufficiently close to the robot to be visible, and $V_{n}^{p}$ are centered i.i.d. random variables that model measurement noise.

To estimate the state $X_{t}=\left(\theta_{t}, x_{t}, p^{1}, \cdots, p^{m}\right)$, one can use an EKF, which is the historical approach to the SLAM problem [14]. Consider then the following (numerical) experiment displayed in Figure 3. The robot starts with approximately known location, e.g. knows in which building it is located, and totally unknown orientation. Using its sensors, it estimates a map of the buliding which consists of 8 priorly unknown features $\left(p^{1}, \cdots, p^{8}\right)$. Staying in the building for sufficiently long, the measurement noise is progressively filtered out, and the relative position between landmarks, that is, the map in the robot's frame, becomes perfectly known although the robot's position and orientation remain uncertain, since the robot does not possess any absolute information. Thus, according to the EKF's covariance matrix, the distance between two landmarks is certain, i.e., it has null (or extremely small) variance. Then, the robot moves out of the building (the Kinect fails to return depth outdoors), and observes the bearing of a visible remote landmark with known location, the Pisa tower (i.e., a typically highly visible landmark, see [27]). Owing to the latter measurement, the robot infers its approximate orientation, and the EKF updates the state $X_{t}$ to take it into account.

As the relative position of unknown features $\left(p^{1}, \cdots, p^{m}\right)$ has been perfectly estimated, it should stay as is, no matter what further measurements are made. However, we see on Figure 4 this is not the case: the distance between two arbitrary features increases according to the EKF when the tower (with known location) becomes visible. This "unphysical" behavior is a problem due to the linear structure of the EKF update that mismatches the nonlinear structure of the dynamics. As can be seen on Figure 4, if in contrast an invariant EKF (IEKF) [1] is used instead, the distance between any two landmarks stays constant no matter what further measurements are made (both filters are identically initialized and tuned).

\subsubsection{Relation to the general theory}

The function we want the filter to preserve is the map in the robot's frame, i.e., $q\left(\theta, x, p^{1}, \cdots, p^{m}\right)=$ $R(\theta)^{T}\left(\left(p^{1}-x\right), \cdots,\left(p^{m}-x\right)\right)$ and the aquired information we want to preserve is thus of the form $q\left(\theta, x, p^{1}, \cdots, p^{m}\right)=b_{t}$ with $b_{t} \in \mathbb{R}^{2 m}$. Moreover, a nonlinear error can be defined as $e=\left(e_{\theta}, e_{x}, e_{p}^{1}, \cdots, e_{p}^{m}\right):=\left(\theta-\hat{\theta}, R(\theta-\hat{\theta})^{T} x-\hat{x}, R(\theta-\hat{\theta})^{T} p^{1}-\hat{p}^{1}, \ldots, R(\theta-\hat{\theta})^{T} p^{m}-\hat{p}^{m}\right)$. We have $q\left(\theta, x, p^{1}, \cdots, p^{m}\right)-q\left(\hat{\theta}, \hat{x}, \hat{p}^{1}, \cdots, \hat{p}^{m}\right)=R(\hat{\theta})^{T}\left(e_{p}^{1}-e_{x}, \cdots, e_{p}^{m}-e_{x}\right)$. Thus the kernel of $D q^{e}$ is the set defined by $e_{x}=e_{p}^{1}=\cdots=e_{p}^{m}$ and it is fixed, so the error is compatible in the sense of Definition 9 and Theorem 1 applies.

The error $e$ is the right-invariant error in the sense of the group $S E_{m+1}(2)$ introduced in $[1,3]$. Thus from Section 5.5 we know the EKF based on error $e$ for the present problem corresponds to the IEKF SLAM of [1].

Alternatively, the results may be applied to an EKF devised using the alternative robot-centered state variables $\left(\theta, R(\theta)^{T} x, R(\theta)^{T}\left(p^{1}-x\right), \ldots, R(\theta)^{T}\left(p^{m}-x\right)\right)$. It is known as the Robocentric mapping EKF [11]. According to Section 4.2, and more precisely Proposition 12 of the Appendix it corresponds to the EKF based on error $e=(\theta-$ 

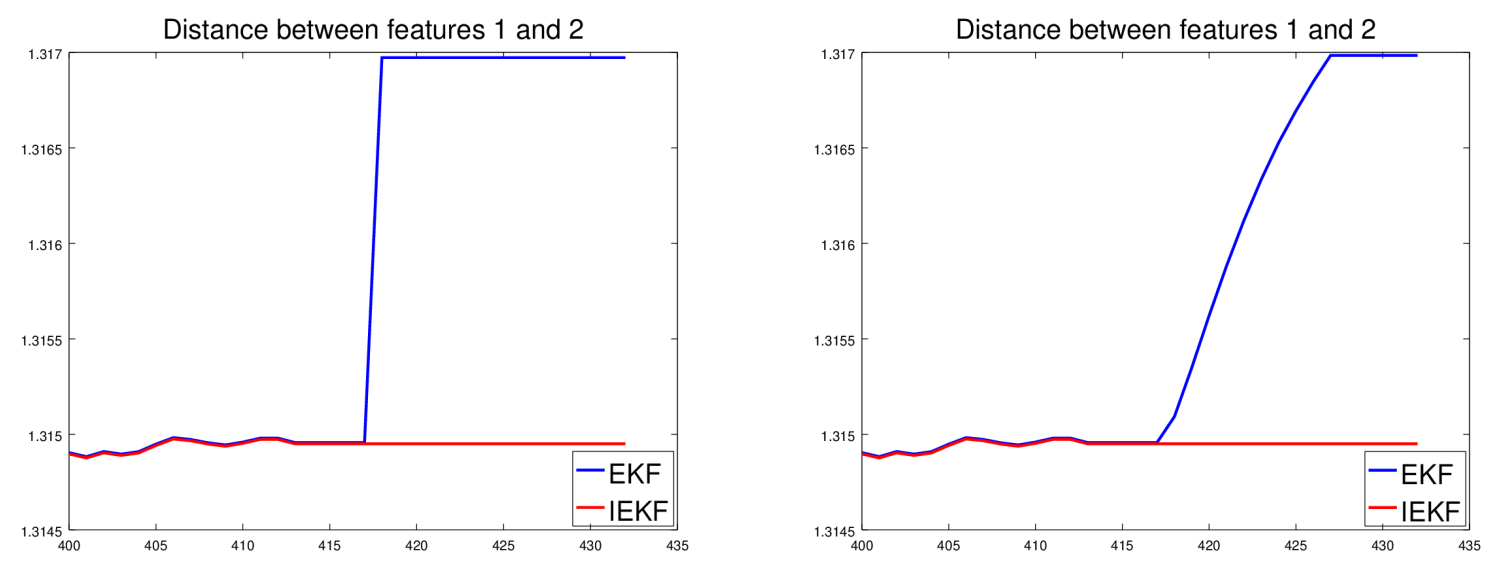

Figure 5: Same experiment as before, where the robot has aquired information about the shape of the map, then goes outdoors and observes Pisa tower whose location is known. Both plots display distance between two arbitrary features between steps 400 and 435 . On the left, a single observation is made at step 417. On the right 10 observations are made from step 417 to 426 with a variance multiplied by 10 (i.e. equivalent total information) to emulate continuous updates. Update frequency has no impact: the discrepancy displayed by the EKF reaches the exact same value and the IEKF preserves the acquired information in both cases.

$\left.\hat{\theta}, R(\theta)^{T} x-R(\hat{\theta})^{T} \hat{x}, R(\theta)^{T}\left(p^{1}-x\right)-R(\hat{\theta})^{T}\left(\hat{p}^{1}-\hat{x}\right), \ldots, R(\theta)^{T}\left(p^{m}-x\right)-R(\hat{\theta})^{T}\left(\hat{p}^{m}-\hat{x}\right)\right)$. It is then straightforward to check Theorem 1 also applies to this filter.

Remark 8. Numerous papers have drawn attention and commented on the caveats of the EKF for SLAM, and notably its problems of consistency see e.g., [17,18]. We would like to emphasize the caveats of the EKF evidenced in the present section are wholly different. The former inconsistencies stem from unobservability of the global map's orientation, whereas the present example is concerned with deterministic information that should be preserved when the problem becomes observable. Note that this is quite relevant to the field of robotics, and can be considered a contribution in itself: the IEKF of [1] and the Robocentric EKF of [11] have just been shown to also resolve another unexplored type of consistency issue of the EKF, related to its unability to produce state updates being consistent with a map previously observed with certainty.

\subsubsection{Case of continous time updates}

One could suspect the differences with a standard EKF are noticeable only in discrete time when an observation creates a large and sudden update which does not occur with high frequency updates. However this is not the case, as proved in the numerical experiment whose results are displayed in Figure 5: if the information arrives in continuous time the induced EKF inconsistency increases progressively but reaches the exact same value as if the same information were provided all at once to the filter. 


\subsection{Extended Kalman filtering with (static) nonlinear equality constraints}

As a byproduct secondary result, the general present theory readily allows enforcing a large range of static nonlinear state equality constraints in $\mathbb{R}^{d}$.

Corollary 2 (Of Theorem 1). Suppose one wants to enforce nonlinear equality constraint $q(X)=c$ with $q: \mathbb{R}^{d} \rightarrow \mathbb{R}^{d-k}$ where $D q(X)$ has constant full rank, and such that $q^{-1}(c):=\mathcal{M}$ is a parallelizable submanifold of $\mathbb{R}^{d}$. Then one can necessarily find an error $e$ which is compatible with $\mathcal{M}$, and the EKF based on $e$ is consistent with the constraint. The estimate thus remains in $\mathcal{M}$, regardless of the measurements.

Error $e$ may be constructively built as follows. If $\mathcal{M}$ is of dimension $k$ and parallelizable there exists smooth vector fields such that $\left\{V_{1}(X), \cdots V_{k}(X)\right\}$ is a basis of $T_{X} \mathcal{M}$ for all $X \in \mathcal{M}$. Consider an error $\Pi(X, \hat{X})$ such that the $k$ first columns of $D \Pi(X)^{-1} \in \mathbb{R}^{d \times d}$ consist of $\left(V_{1}(X), \cdots V_{k}(X)\right)$, and the $d-k$ remaining columns are a basis of $\operatorname{Im} D q(X)$. This error is compatible: for $X \in \mathcal{M}$, Ker $D q(X)=\operatorname{Im}\left(V_{1}(X), \cdots V_{k}(X)\right)$ and thus $\operatorname{Ker} D q^{e}(X)=\operatorname{Ker} D q(X)(D \Pi(X))^{-1}$ is fixed (spanned by the first $k$ columns of $I_{d \times d}$ ) and Theorem 1 applies. Note that, with process noise, the results still hold along the lines of Section 4.4.

Extended Kalman filtering on Lie groups has been the subject of recent research. Indeed, one may want the estimate to remain on the group, such as in e.g., $[9,10,15]$, and this is one type of (static) constraint one may consider, albeit far less challenging than the time-varying subgroup constraints of Section 5 .

For instance for attitude estimation, if $X \in \mathbb{R}^{4}$, and $*$ denotes the quaternion product extended in a straightforward way to all vectors of $\mathbb{R}^{4}$, then the EKF based on error $e=X^{-1} * \hat{X}$ automatically enforces $\|X\|=1$ if correctly initialized, and thus that $X$ is a quaternion. Indeed for $q(X)=\|X\|^{2}$, Ker $D q^{e}$ is spanned by vectors whose first coordinate is 0 , and is thus fixed. The error is thus compatible and Theorem 1 applies. Note that, the alternative error $e=\hat{X} * X^{-1}$ leads to identical results. See also the recent work of [15] on the subject.

\section{Conclusion}

In this paper we have proposed a general methodology of EKF based on nonlinear errors. We have proved it may be applied to the problem of nonlinear filtering in the presence of partial deterministic information, i.e., constraints, in order to have the EKF account for this information, and not violate it as is the case with the conventional EKF. Given the constraints, there is no systematic way to construct the appropriate error, but given an error, Proposition 8 provides a simple way to check its compatibility with the constraints. The special case where the state space is a Lie group, was shown to come with much stronger results. Finally, we have provided a novel application to SLAM.

A very important point is as follows. Our method consists in using alternative EKFs, not in artificially constraining EKFs. As a result, there is a continuum between the constrained case and the non-constrained case in our theory, which is not the case of stateconstrained solutions. This is a strong indication that our EKF will perform well in the presence of small, albeit not necessarily null, eigenvalues in the covariance matrix. This 
is illustrated by our SLAM example, where in simulations eigenvalues in the covariance matrix actually become very small due to multiple observations, but not strictly null, and the desired behavior is yet achieved. This is also key to the success of the industrial application of IEKF to inertial navigation as described in [6], where the dynamics are close to being noise-free, owing to high precision inertial sensors, but not completely noise-free as sensors always come with (small) noises that engineers do account for in the process noise covariance matrix $Q$ of the IEKF.

As a perspective, one could study the benefits of the present theory in the context of data assimilation, and attempt to extend some linear results of $[8,16,21]$ to the nonlinear case.

\section{References}

[1] A. Barrau and S. Bonnabel. An EKF-SLAM algorithm with consistency properties. Technical report. arXiv:1510.06263, 2015.

[2] A. Barrau and S. Bonnabel. Navigating with highly precise odometry and noisy GPS: a case study. IFAC-PapersOnLine, 49(18):618-623, 2016.

[3] A. Barrau and S. Bonnabel. The invariant extended Kalman filter as a stable observer. IEEE Transactions On Automatic Control, 62(4):1797-1812, 2017.

[4] A. Barrau and S. Bonnabel. Three examples of the stability properties of the invariant extended Kalman filter. IFAC-PapersOnLine, 50(1): 431-437, 2017.

[5] A. Barrau and S. Bonnabel Intrinsic filtering on $\mathrm{SO}(3)$ with discrete-time observations. In Conference on Decision and Control (CDC), 2012, pages 3255-3260. IEEE, 2013.

[6] A. Barrau and S. Bonnabel. Invariant Kalman filtering. Annual Review of Control, Robotics, and Autonomous Systems, Vol. 1:237-257, 2018.

[7] B. M Bell, J. V Burke, and G. Pillonetto. An inequality constrained nonlinear Kalman-Bucy smoother by interior point likelihood maximization. Automatica, 45(1):25-33, 2009.

[8] M. Bocquet, K. S Gurumoorthy, A. Apte, A. Carrassi, C. Grudzien, and C. KRT Jones. Degenerate Kalman filter error covariances and their convergence onto the unstable subspace. SIAM/ASA Journal on Uncertainty Quantification, 5(1):304333, 2017.

[9] J. Bohn and A. K Sanyal. Unscented state estimation for rigid body motion on SE(3). In Conference on Decision and Control (CDC), 2012, pages 7498-7503. IEEE, 2012.

[10] G. Bourmaud, R. Mégret, M. Arnaudon, and A. Giremus. Continuous-discrete extended Kalman filter on matrix Lie groups using concentrated Gaussian distributions. Journal of Mathematical Imaging and Vision, 51(1):209-228, 2015. 
[11] J. A Castellanos, R. M. Cantin, J. D Tardós, and J. Neira. Robocentric map joining: Improving the consistency of EKF-SLAM. Robotics and autonomous systems, $55(1): 21-29,2007$.

[12] P. Chauchat, A. Barrau, and S. Bonnabel. Kalman filtering with a class of geometric state equality constraints. In IEEE 56th Conference on Decision and Control (CDC), 2017.

[13] P. E. Crouch, A. J. van der Schaft Variational and Hamiltonian control systems. In Springer-Verlag New York, 1987.

[14] H. Durrant-Whyte and T. Bailey, Simultaneous localization and mapping: part I, IEEE Robotics Automation Magazine, 13(2):99-110,2006.

[15] J. R. Forbes, A. HJ de Ruiter, and D. E. Zlotnik. Continuous-time norm-constrained Kalman filtering. Automatica, 50(10):2546-2554, 2014.

[16] K. S Gurumoorthy, C. Grudzien, A. Apte, A. Carrassi, and C. KRT Jones. Rank deficiency of Kalman error covariance matrices in linear time-varying system with deterministic evolution. SIAM Journal on Control and Optimization, 55(2):741-759, 2017.

[17] G. P. Huang. Towards consistent filtering for discrete-time partially-observable nonlinear systems. Systems \& Control Letters, 106:87-95, 2017.

[18] G. P. Huang, A. I. Mourikis, and S. I. Roumeliotis. Observability-based rules for designing consistent EKF SLAM estimators. International Journal on Robotics Research (IJRR), 29(5):502-528, April 2010.

[19] A. Khosravian, J. Trumpf, R. Mahony, and T. Hamel. State estimation for invariant systems on Lie groups with delayed output measurements. Automatica, 68:254-265, 2016 .

[20] J. M. Lee Introduction to smooth manifolds, Springer. 2001.

[21] P. Moireau, D. Chapelle, and P. Le Tallec. Joint state and parameter estimation for distributed mechanical systems. Computer methods in applied mechanics and engineering, 197(6-8):659-677, 2008.

[22] B. Ni and Q. Zhang. Stability of the Kalman filter for continuous time output error systems. Systems \& Control Letters, 94:172-180, 2016.

[23] D. Simon. Kalman filtering with state constraints: a survey of linear and nonlinear algorithms. IET Control Theory $\&$ Applications, 4(8):1303-1318, 2010.

[24] D. Simon and T. L. Chia. Kalman filtering with state equality constraints. IEEE transactions on Aerospace and Electronic Systems, 38(1):128-136, 2002.

[25] J. Stillwell. Naive Lie Theory. Springer, 2008. 
[26] G. Teschl. Ordinary Differential Equations and Dynamical Systems. Americal Mathematical Society, 2012.

[27] S. Thrun, W. Burgard, and D. Fox. Probabilistic robotics. MIT press, 2005.

\section{Appendix A: Change of variables}

Proposition 12. Let $\phi: \mathbb{R}^{d} \rightarrow \mathbb{R}^{d}$ be a diffeomorphism encoding a change of variables. Let $Z=\phi(X)$. Then, the EKF based on the error variable $e=\Pi(\hat{X}, X):=\phi(X)-\phi(\hat{X})$, that is, the standard linear error $Z-\hat{Z}$ in the alternative variable $Z$, coincides with the conventional EKF built using variable $Z$. More precisely if $\hat{X}_{t}, P_{t}^{e}$ are the parameters output by the EKF based on error e, and if $\hat{Z}_{t}, P_{t}^{Z}$ are those output by the conventional EKF built using the $Z$-coordinates system, then we have at all times $\hat{Z}_{t}=\phi\left(\hat{X}_{t}\right)$ and $P_{t}^{e}=P_{t}^{Z}$, as soon as initially $\hat{Z}_{0}=\phi\left(\hat{X}_{0}\right)$ and $P_{0}^{e}=P_{0}^{Z}$.

Proof. In this case we have $D \Pi(\hat{X})=D \phi(\hat{X})$. The propagation step obviously preserves that $\hat{Z}_{t}=\phi\left(\hat{X}_{t}\right)$, since it consists of a mere integration of the dynamics. To prove it preserves $P_{t}^{e}=P_{t}^{Z}$ we first need to prove the equality of the linearized systems, i.e., that $A_{t}^{e}=A_{t}^{Z}$. We will skip this step, which can easily be established similarly to (26). As concerns the update step, the proof is based on the following technical result:

Lemma 2. $\phi \circ \varphi(x, \delta e)=\phi(x)+\delta e$ for all $x, \delta e$.

Proof. $\varphi(x, \delta e)$ is defined as the solution $\tilde{\chi}(1)$ at $s=1$ to $\frac{d}{d s} \tilde{\chi}(s)=[D \phi(\tilde{\chi}(s))]^{-1} \delta e=$ $D \phi^{-1}(\phi(\tilde{\chi}(s))) \delta e$. But $\frac{d}{d s} \phi^{-1}(\phi(x)+s \delta e)=D \phi^{-1}(\phi(x)+s \delta e) \delta e$. As $\phi(\tilde{\chi}(0))=\phi(x)$ we have $\phi(\tilde{\chi}(s))=\phi(x)+s \delta e$ or all $s \geq 0$, proving $\tilde{\chi}(1)=\phi(x)+\delta e$.

In the $Z$ coordinates, the linearized output map writes $H_{n}^{Z}=\frac{\partial}{\partial z} h \circ \phi^{-1}(\hat{Z})=$ $H_{n} D \phi^{-1}(\phi(\hat{X}))$ where $H_{n}=\frac{\partial}{\partial x} h(\hat{X})$, and using $(22)$ and $(15) H_{n}^{e}=H_{n}(D \phi(\hat{X}))^{-1}=H_{n}^{Z}$, yielding identical $K_{n}, P_{t_{n}}^{+}$, and $K_{n} z_{n}$ for both filters. Using Lemma 2 , we then see that both filters also yield the same updated state.

\section{Appendix B : Proof of Theorem 5}

Lemma 3. The propagation step of the EKF based on error $e_{t}$ in the sense of Section 4, writes:

$$
\frac{d}{d t} \hat{\chi}_{t}=f_{u_{t}}\left(\hat{\chi}_{t}\right), \quad \frac{d}{d t} P_{t}^{e}=A_{t}^{e} P_{t}^{e}+P_{t}^{e}\left(A_{t}^{e}\right)^{T},
$$

where $A_{t}^{e}$ is defined at Theorem 3. Moreover, (38) is the propagation step of the LIEKF of [3].

Proof. $A_{t}^{e}$ represents the first-order approximation to the error evolution. Let us apply the methodology of Section 4 using the first-order geometry of $G$, see Remark 5 . Indeed, according to (29) and (30) the full error equation during propagation is $\frac{d}{d t} e_{t}=g_{u_{t}}\left(e_{t}\right)$. To linearize the error around the diagonal $\Pi(\chi, \chi)=I$, we posit $e_{t}=\exp \left(\delta e_{t}\right)$ with $\delta e_{t} \in \mathbb{R}^{d}$. Remarkably, the evolution of $\xi_{t}$ needs not be linearized as Theorem 3 proves there is $A_{t}^{e} \in \mathbb{R}^{d \times d}$ indeed, defined as $d\left(g_{u_{t}}\right)_{I}$, such that $\frac{d}{d t} \delta e_{t}=A_{t}^{e} \delta e_{t}$, and where $\exp \left(\delta e_{t}\right)$ 
is exactly $e_{t}$. As this is true in particular at the first order, (38) corresponds indeed to the propagation step (17) of the EKF based on $e_{t}$. And it turns out this is exactly the definition of the propagation step of the LIEKF of [3].

Lemma 4. Under conditions of Theorem 5, the update step of the EKF based on error $e_{t}$ in the sense of Section 4 writes:

$$
\begin{aligned}
z_{n} & :=Y_{n}-\hat{\chi}_{t_{n}} \cdot \bar{d}, \\
K_{n} & :=P_{t_{n}}^{e}\left(H_{n}^{e}\right)^{T}\left(H_{n}^{e} P_{t_{n}}^{e}\left(H_{n}^{e}\right)^{T}+R_{n}\right)^{-1}, \\
\hat{\chi}_{t_{n}}^{+} & =\hat{\chi}_{t_{n}} \exp \left(K_{n} z_{n}\right), \quad\left(P_{t_{n}}^{e}\right)^{+}=\left(I-K_{n} H_{n}^{e}\right) P_{t_{n}}^{e},
\end{aligned}
$$

with $H_{n}^{e}: \mathbb{R}^{d} \mapsto \mathbb{R}^{N}$ the linear map defined by $\delta e \mapsto \hat{\chi}_{t_{n}}(\delta e)^{\wedge} \bar{d}$. Moreover, this exactly coincides with the update step of the LIEKF of [3].

Proof. Regarding the definition of $\hat{\chi}_{t_{n}}^{+}$in (41), we used (20) and the result (36). In the methodology of Section $4, H_{n}^{e}$ is defined as the differential of $h$ with respect to error $e$. As noticed, this means on $G$ that $h(\chi)-h\left(\hat{\chi}_{t_{n}}\right)=H_{n}^{e} \delta e+O\left(\|\delta e\|^{2}\right)$ where $\Pi\left(\hat{\chi}_{t_{n}}, \chi\right)=\exp \delta e$, that is, $\chi=\hat{\chi}_{t_{n}} \exp (\delta e)$. Assuming $h\left(\hat{\chi}_{t_{n}} \exp (\delta e)\right)=\hat{\chi}_{t_{n}} \exp (\delta e) \cdot \bar{d}$, a first-order expansion of the exponential yields $H_{n}^{e} \delta e=\hat{\chi}_{t_{n}}(\delta e)^{\wedge} \bar{d}$.

The seemingly different update of the LIEKF [3] is:

$$
\begin{aligned}
\tilde{K}_{n} & =P_{t_{n}}^{e}(H)^{T}\left(H P_{t_{n}}^{e} H^{T}+\hat{R}_{n}\right)^{-1}, \\
\hat{\chi}_{t_{n}}^{+} & =\hat{\chi}_{t_{n}} \exp \left[\tilde{K}_{n}\left(\hat{\chi}_{t_{n}}^{-1} Y_{n}-\bar{d}\right)\right], \\
\left(P_{t_{n}}^{e}\right)^{+} & =\left(I-\tilde{K}_{n} H\right) P_{t_{n}}^{e},
\end{aligned}
$$

with $H: \delta e \mapsto(\delta e)^{\wedge} \bar{d}$, and $\hat{R}_{n}=\hat{\chi}_{t_{n}}^{-1} R_{n}\left(\hat{\chi}_{t_{n}}^{-1}\right)^{T}$. But it is easily seen that $H_{n}^{e}=\hat{\chi}_{t_{n}} H$, that $K_{n}=\tilde{K}_{n}\left(\hat{\chi}_{t_{n}}\right)^{-1}$ and thus both steps exactly coincide. 